\title{
18. ESTIMATING LITHOLOGY FROM NONINTRUSIVE REFLECTANCE SPECTRA: LEG 138 ${ }^{1}$
}

\author{
Alan C. Mix, ${ }^{2}$ Sara E. Harris, ${ }^{2}$ and Thomas R. Janecek ${ }^{3}$
}

\begin{abstract}
During Leg 138, we measured reflectance spectra in the visible and near-infrared bands ( $455-945 \mathrm{~nm}$ ) every few centimeters on split core surfaces from eastern tropical Pacific Ocean sediments. Here, we evaluate predictions of the content of biogenic calcite, biogenic opal, and nonbiogenic sediments from the reflectance spectra. For Sites 844 through 847, which contain a significant nonbiogenic component, reflectance spectra yielded a useful proxy for the percentages of $\mathrm{CaCO}_{3}$ over a wide range of values from nearly $0 \%$ to $100 \%$, with root-mean-square (RMS) errors of about $9 \%$. Direct estimates of "nonbiogenic" sediment percentages, approximated by $100-\left(\mathrm{CaCO}_{3}+\right.$ opal), were reasonably successful (RMS error of $\left.10 \%\right)$, however, were incorrect in some intervals. This suggests that mineralogy of the nonbiogenic material changes through time and that further subdivision of this component will be needed for useful estimation from reflectance. For percentages of biogenic opal, calibration equations appear to work well (RMS error of 6\%) at concentrations of less than $30 \%$, but for higher opal concentrations, reflectance equations often underestimate the true contents of opal. Improvements in multiparameter lithologic estimates from reflectance spectra may come from (1) expanding the wavelengths measured to better capture unique mineral reflectance bands, and (2) adding the ability to measure diffuse, rather than directional, reflectance to minimize the effects of surface roughness.
\end{abstract}

\section{INTRODUCTION}

This study assesses the utility of reflectance spectroscopy as a tool for rapid, nondestructive analysis of deep-sea sediments. A tool such as this offers great potential for cost-effective analysis of long time series of lithologic change at high temporal resolution. Previous attempts to measure lithology from reflectance in ODP cores considered only gray-scale optical density, as recorded in black-and-white photographs (Busch, 1991). Initial success of that study motivated us to measure reflectance in an array of wavelengths as a way of isolating lithologic components. Balsam and Deaton (1991) and Balsam (1992) showed that reflectance spectra in the near-ultraviolet (nUV), visible (VIS), and near-infrared (nIR) bands, measured on discrete samples that have been carefully dried, ground, and mounted, can be calibrated to yield quantitative estimates of some aspects of lithology in deepsea sediments.

Our contribution (Mix et al., 1992) was the development of a reflectance spectrometer system that automatically scans split core sections rapidly at closely spaced intervals. Clark et al. (1990) showed that spectrometers having high spectral resolution (on the order of 1 $\mathrm{nm}$ ) are best suited for reliable recognition of minerals, which guided our design of an instrument suitable for use on deep-sea sediments. The Oregon State University (OSU) instrument used during Leg 138 measured reflectance spectra in 511 data channels that cover most of the VIS (455-700 nm) and nIR (700-945 nm) bands at high spectral resolution (bandwidth, $0.96 \mathrm{~nm}$ ) fast enough (about $10 \mathrm{~s}$ per sample) to keep up with ODP coring operations. The instrument automatically analyzed reflectance spectra every few centimeters in more than $5 \mathrm{~km}$ of sedimentary sections recovered with the advanced piston corer (APC) and extended core barrel (XCB) devices in the eastern tropical Pacific Ocean. Based on shipboard results, we found that this tool helped to constrain the development of composite depth sections as cores were collected (Hagelberg et al., 1992) and yielded qualitative insight into sediment composition (Mix et al., 1992).

' Pisias, N.G., Mayer, L.A., Janecek, T.R., Palmer-Julson, A., and van Andel, TH (Eds.), 1995. Proc. ODP, Sci. Results, 138: College Station, TX (Ocean Drilling Program)

${ }^{2}$ College of Oceanic and Atmospheric Sciences, Oregon State University, Corvallis, OR 97331-5503, U.S.A.

${ }^{3}$ Ocean Drilling Program, Texas A \&M University Research Park, 1000 Discovery Drive, College Station, TX 77845-9547, U.S.A.
Here, we calibrate the reflectance data from the OSU reflectance spectrometer to yield quantitative estimates of lithology. We show that even when analyzing unprepared core surfaces, one can estimate some lithologic components with sufficient precision to yield useful quantitative geologic reconstructions. This study is preliminary. It points the way to further development of the surface scanning reflectance spectrometer and data analysis techniques to optimize the recovery of high-resolution lithologic information from this rapid remote sensing strategy.

One might ask why bother with reflectance? Wet bulk density was measured in the same samples by GRAPE (gamma ray attenuation porosity evaluator; Boyce, 1976), and this is often considered a good proxy for $\mathrm{CaCO}_{3}$ contents in pelagic sediments (Mayer, 1991; Hagelberg et al., this volume). The transformation of GRAPE density into percentages of $\mathrm{CaCO}_{3}$, however, depends on constancy of the wet-bulk density of the sediments diluting the calcium carbonate, as well as the grain size of the carbonate and noncarbonate components, porosity rebound, and compaction (Mayer, 1991). Where the sediment is a simple binary mixture of biogenic opal and biogenic carbonate, GRAPE is an excellent proxy for calcium carbonate contents. Where a third sedimentary component of different density and porosity (such as clay or other nonbiogenic material) is present, the GRAPE-to-calcite transform is less certain (Herbert and Mayer, 1991). This is the case in the eastern transect sites of Leg 138 (Sites 844-847). Thus, we concentrated our analysis on these eastern sites (Fig. 1), where our primary goal was to improve estimates of $\mathrm{CaCO}_{3}$ percentages.

Reflectance spectroscopy is an independent approach having different assumptions, strengths, and weaknesses than GRAPE. In some cases, reflectance may be a better tool, such as for XCB cores, in which the sediments break into oblong "biscuits" within the core liners. These biscuits are damaging to the GRAPE method, because water-saturated slurry often fills the spaces between biscuits along the core edges, and GRAPE measurements integrate a finite slice across the complete core cross section. Reflectance spectroscopy is less sensitive to this problem, because sediment biscuits tend to meet along the axis of a core tube, where the reflectance is measured. A possible weakness of the reflectance method is its potential sensitivity to surface roughness and moisture content of unprepared surfaces. Thus, a second goal was to evaluate the severity of these problems for both GRAPE and reflectance methods in typical deep-sea sediments by assessing the precision of quantitative lithologic estimates, compared to ground-truth measurements. Finally, reflectance spectro- 


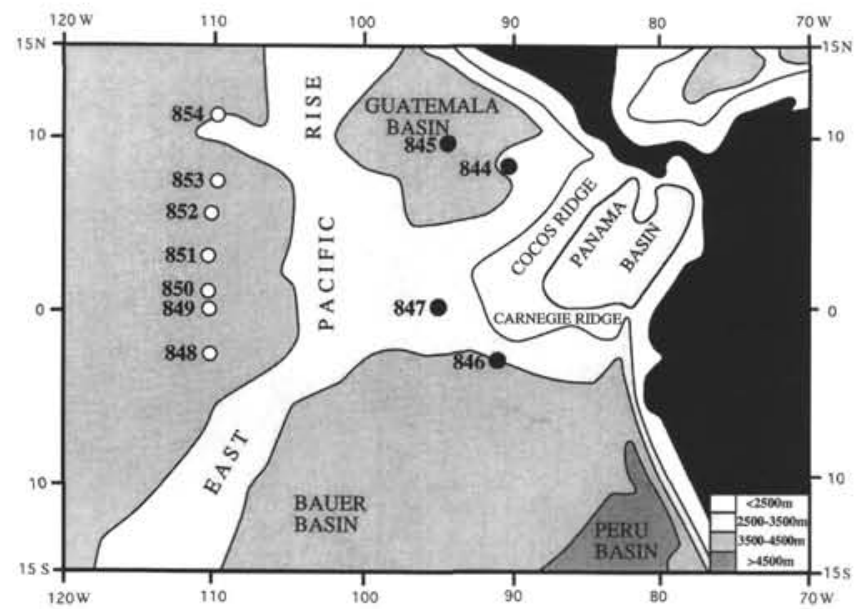

Figure 1. Leg 138 sites. Solid circles mark eastern transect sites (844-847) considered in this study.

scopy offers the potential to estimate several different lithologic parameters from the same spectral data set, should different mineral assemblages have different reflectance signatures. We consider this potential, concentrating here on the major sediment components calcium carbonate, opal, and nonbiogenic (mostly clay) sediments.

\section{REFLECTANCE DATA}

Reflectance spectra were measured at sea with a prototype instrument described in detail by Mix et al. (1992). The instrument consists of a core transport system, an illumination and sensing system, a spectrograph and detector system, and a computer control and data processing system. Similar to the ODP multisensor track, the core transport, or conveyor, moves a $1.5-\mathrm{m}$ core section under the light source and detector at fixed intervals (usually 3 or $4 \mathrm{~cm}$ ). A regulated broadband light source illuminates a spot $2-\mathrm{cm}$ in diameter on a split core surface, through glass fiber-optic cables. Light is delivered and collected by a custom-designed sensing head, which hovers a fixed distance above the sediment surface. The sensing head collects directional reflectance along an axis perpendicular to the core surface. Reflected light is transferred back to the spectrograph, again through fiber-optic cables. Separation of the reflected light into its spectrum occurs in the spectrograph, which has an entrance slit, diffraction grating, focusing mirrors, and a silicon diode array detector. The computer control system consists of a 386-PC to control the mechanical system and detector, a Sun SLC UNIX workstation to receive and process the data, and a $600-\mathrm{Mb}$ disk and $150-\mathrm{Mb}$ tape drive to store the data.

We measured directional reflectance in 511 different wavelengths of light that ranged from about 455 to $945 \mathrm{~nm}$ (most of the VIS and the lower portions of the nIR bands). At each site, the standard core description included a down-core plot of three 50-nm blocks of reflectance channels (blue, red, and near-infrared), and examples of fullresolution 511-channel reflectance spectra from distinctive lithologies (Fig. 2). A preliminary look at the reflectance spectra showed that different major lithologies, such as nannofossil ooze, diatom ooze, or siliceous clay, exhibit radically different spectra (Mix et al., 1992). In addition, minor components, such as oxides, impart distinctive features to the reflectance spectra.

Some analytical noise was present in the spectra of Figure 2, especially near the low- and high-wavelength limits of the spectra. With the prototype instrument used during Leg 138, a tradeoff was made between speed of analysis and noise. To keep up with coring operations, we set short signal integration times. Typical analyses took $2.5 \mathrm{~s}$. Each measurement was replicated three times, and these replicates were averaged. The total analysis time, including replicate analyses, core movement, focusing, and data storage, took about $10 \mathrm{~s}$. New developments in CCD detector array technology will reduce the noise and increase the speed of analysis in the next generation of instruments. For purposes of calibrating the reflectance values to predict lithologies for this preliminary study, we smoothed the reflectance spectra by taking 20 -channel block averages, starting with the longest wavelengths. This reduced the 511 channels to 25 channels, with a $19.2 \mathrm{~nm}$ bandwidth for use in the regression analyses.

The features we see in the Leg 138 spectra and lithologies are qualitatively consistent with features expected from laboratory experiments on pure mineral separates and known mineral mixtures (Hunt, 1977; Clark et al., 1990). For example, sediments high in calcium carbonate are visually "white" and are highly reflective in all bands measured. This is consistent with analyses of reagent calcite, which reflects close to $100 \%$ in the VIS and nIR bands (Gaffey, 1986). Nonbiogenic and biogenic calcite can differ in their reflectance character; however, in the sediments considered here, essentially all of the carbonate material is biogenic calcite.

Minor components may be important to reflectance characteristics of sediments. Oxides (commonly hematite) make the sediments appear reddish brown and are easily detected in the reflectance spectra, as shown by Deaton and Balsam (1991). Although both biogenic opal and calcite are highly reflective throughout the VIS and nIR bands measured here, they diverge in their spectra at wavelengths of less than about $500 \mathrm{~nm}$ (Balsam, 1992). Sulfides, such as pyrite, had relatively low reflectance throughout the bands we measured (Hunt et al., 1971b). Organic carbon is a relatively minor constituent of the sediment, but may have significant effects on reflectance (Busch, 1991) because it absorbs light in the visible bands (Hoffer, 1984; Baker and Louda, 1986), and because of its role in sedimentary redox chemistry, which controls the balance of oxides and sulfides (Lyle, 1983). The presence of water can affect the reflectance spectra and, thus, calibration of equations to predict lithology from the surface scanned data collected here must be done with samples that contain water (Clark, 1981).

\section{GROUND-TRUTH DATA}

Our calibration strategy was empirical. We sought significant relationships between "ground-truth" chemical measurements of sediment composition and accompanying reflectance spectra. For this strategy to work, the calibration data set must span the range of variation in the samples to be estimated, including the possible combinations of different lithologies. If more than one lithologic parameter is to be estimated, the various parameters must not correlate highly with each other in the calibration data set.

We calibrated reflectance spectra to predict percentages of the dominant sedimentary components, $\mathrm{CaCO}_{3}$, opal, and nonbiogenic sediments using standard multiple regression techniques. The groundtruth samples analyzed chemically were taken at sea for measuring physical properties, usually one sample per 1.5 - $\mathrm{m}$ section, from almost all cores recovered. Thus, the data set was a reasonable random sampling of a broad range of sediment types recovered from Sites 844 through 847 during Leg 138. All calibration and verification samples are from APC cores, chosen to avoid problems of lithification and disturbance in the XCB cores.

Concentrations of $\mathrm{CaCO}_{3}$ (essentially all biogenic calcite) were measured at sea (Mayer, Pisias, Janecek, et al., 1992) using a Coulometrics $5011 \mathrm{CO}_{2}$ coulometer equipped with a System 140 carbonate carbon analyzer. A 10 - to 60-mg dry sample was reacted in a solution of $2 \mathrm{~N} \mathrm{HCl}$ while it was heated at $65^{\circ} \mathrm{C}$. The evolved $\mathrm{CO}_{2}$ was transferred to the $\mathrm{CO}_{2}$ coulometer cell in a helium stream. The quantity of $\mathrm{CO}_{2}$ liberated was measured by titration in a monoethanolomine solution with a proprietary colorimetric indicator. The change in light transmittance was monitored by a photodetection cell. Reagent-grade calcite was used as a standard, and the percentage of $\mathrm{CaCO}_{3}$ was 
Reflectance 650-700nm (\%)
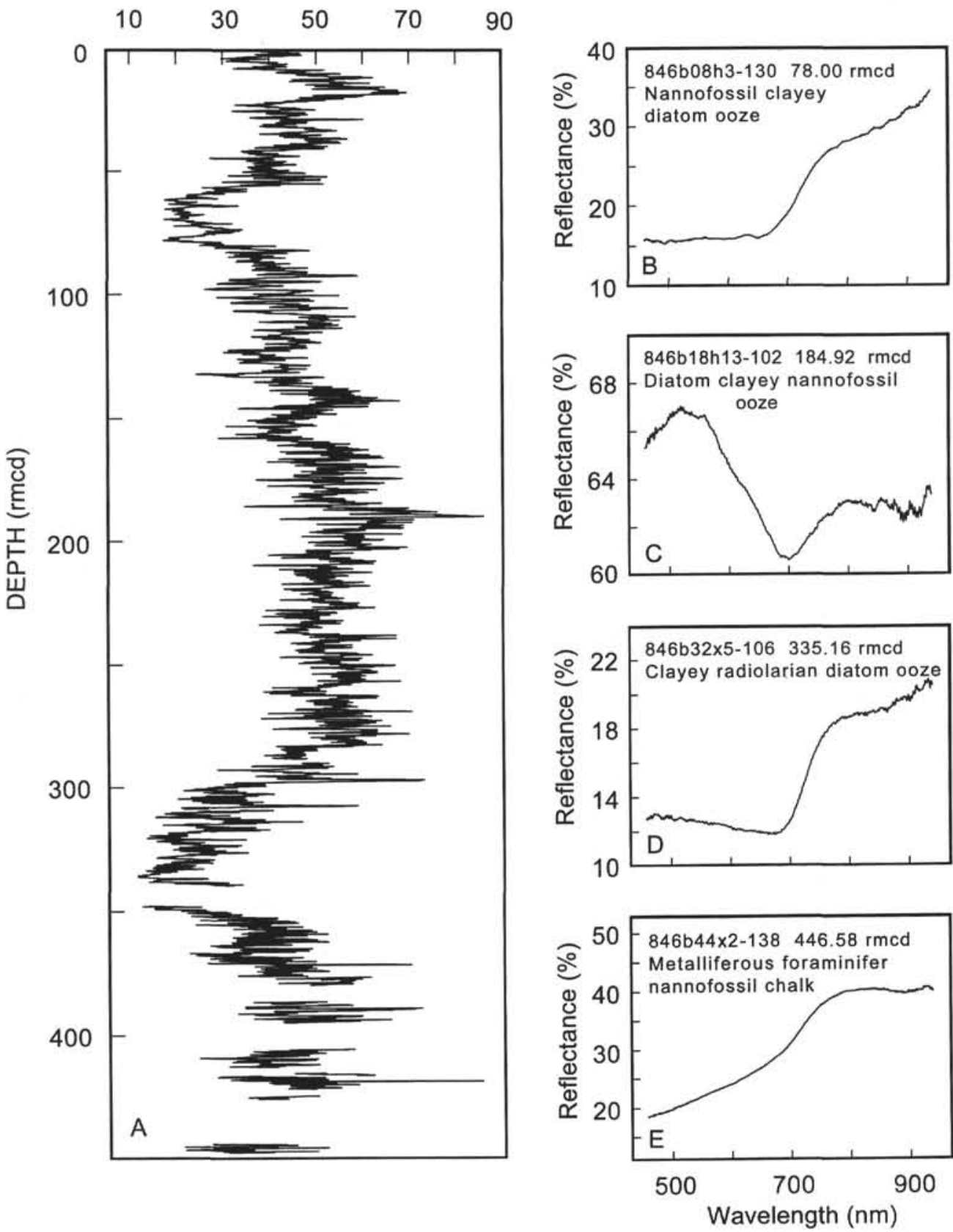

Figure 2. A. Percentage of reflectance in the 700- to 750-nm "red" band, in a composite section constructed by stacking and averaging analyses in Holes 846A through 846D. Depths are revised meters composite depth (rmcd), which seek to align features in all holes at the site (Hagelberg et al., this volume). B-E. Examples of reflectance spectra from individual samples for selected lithologies downhole. Spectra plotted are 11-channel running averages, for an effective bandwidth of $\sim 10 \mathrm{~nm}$. Note the similarity of the two diatom ooze samples ( $\mathbf{B}$ and $\mathbf{D}$ ) compared to the different reflectance spectra for the nannofossil ooze sample $(\mathbf{C})$ and metalliferous chalk $(\mathbf{E})$.

calculated from the inorganic carbon concentration (IC), assuming that all carbonate occurred as $\mathrm{CaCO}_{3}$, as follows:

$$
\mathrm{CaCO}_{3} \%=\mathrm{IC} \% \cdot 8.334 \text {. }
$$

Duplicate analyses performed during Leg 138 indicated excellent reproducibility, with an RMS error of $0.8 \%$ for $\mathrm{CaCO}_{3}$ percentages.

Percentages of opal (essentially all biogenic) were determined at the Ocean Drilling Program, College Station, TX, using the wet- alkaline extraction procedure of Mortlock and Froelich (1989). A sample of 25- to 200-mg dry sediment was subjected to successive extractions with acid and peroxide to remove diluting phases (such as carbonate minerals and organic matter). Biogenic silica then was extracted for $5 \mathrm{hr}$ in a $2 \mathrm{M} \mathrm{Na}_{2} \mathrm{CO}_{3}$ solution in an $85^{\circ} \mathrm{C}$ water bath. The dissolved silica concentration in the extract was determined by molybdate-blue spectrometry using an absorption peak at $812 \mathrm{~nm}$. Precision of this method is better than $0.1 \%$ for opal. 
Nonbiogenic sediment concentration in the ground-truth samples was calculated here as:

$$
\text { Nonbiogenic sediment } \%=100-\left(\text { opal } \%+\mathrm{CaCO}_{3} \%\right) \text {. }
$$

Analytically, this value is not independent of the other two measurements. Although this calculation is only approximate, we think that this simple subdivision of the bulk sediment into its major components is reasonable for a first attempt at lithologic calibration. Future efforts should subdivide the nonbiogenic category into its constituents and may revise the preliminary view given here.

The three lithologic parameters span a wide range of variation in the ground-truth data set (Fig. 3). Percentages of $\mathrm{CaCO}_{3}$ range from $0 \%$ to $90 \%$. Opal concentrations range from $0 \%$ to $70 \%$. Nonbiogenic sediment percentages range from $0 \%$ to $90 \%$. For an ideal calibration, each of the three components should not correlate with the others. Here, the calibration and verification data sets approximate this condition for opal and nonbiogenic sediment concentrations $(r=0.04, n=$ 398). This is also the case for percentages of $\mathrm{CaCO}_{3}$ and opal $(r=$ $-0.25, n=398$ ). Unfortunately, this is not the case for concentrations of $\mathrm{CaCO}_{3}$ and nonbiogenic sediment. These two parameters are strongly inversely correlated $(r=-0.98, n=398)$. Thus, we cannot consider the equations that estimate $\mathrm{CaCO}_{3}$ and nonbiogenic sediment content to be statistically independent of each other.

For purposes of developing and testing equations to estimate lithology from reflectance, the ground-truth data were divided into two groups: a calibration data set (used in the multiple regression) and a verification data set (used to check the resulting equation). To divide the data, we assembled all the analyses in order of depth within each hole and sorted odd-numbered samples into the calibration data set and even-numbered samples into the verification data set. This yielded two independent data sets of approximately 200 samples each, with similar means and distribution of variance. By showing that the precision of estimate was good in a verification data set, we gained confidence that the statistical model was not overly tuned and that the lithologic predictions were robust. The calibration and verification data sets are documented here in Tables 1 and 2. All reflectance data generated by Mix et al. (1992) have been tabulated on CD-ROM, as part of the Leg 138 shipboard data report in Mayer, Pisias, Janecek, et al. (1992).

\section{EMPIRICAL CALIBRATIONS}

\section{Biogenic Calcium Carbonate}

Figure 4 illustrates preliminary calibrations of reflectance spectra to estimate percentages of $\mathrm{CaCO}_{3}$. In this multiple regression analysis, we entered terms for linear reflectance and the square of reflectance in each band to allow for weak nonlinearity. We kept only terms above a $95 \%$ confidence interval. The resulting equation for estimation of carbonate percentages is as follows:

$$
\begin{gathered}
\mathrm{CaCO}_{3} \%=8.42\left(R_{530}\right)-7.14\left(R_{550}\right)-8.37 \times 10^{-3}\left(R_{570}\right)^{2}+2.94\left(R_{800}\right) \\
-2.21 \times 10^{-2}\left(R_{935}\right)^{2}-63,
\end{gathered}
$$

where $R$ is the average reflectance within a $20-\mathrm{nm}$ bandwidth centered on the subscripted wavelength. The equation has $r^{2}=0.89$, meaning that it reproduces $89 \%$ of the variance in the calibration data set. The RMS error on percentages of $\mathrm{CaCO}_{3}$ estimated with this equation is $\pm 9.3 \%$ over the full range of values from $<5 \%$ to $>90 \%$. The errors in the carbonate prediction were similar in the calibration data set and the verification data set (Figs. $4 \mathrm{~A}$ and $4 \mathrm{~B}$ ), and the verification data set has $r^{2}=0.88$. Residuals (predicted values minus measured values) were randomly distributed relative to the measured values. The estimate of $\mathrm{CaCO}_{3}$ percentages from reflectance in these samples is statistically significant and appears to be unbiased and robust.

Because pure calcite is highly reflective in all bands measured here (Hunt and Salisbury, 1971; Gaffey, 1986), the reflectance calibration equation responds mostly to variations in the noncarbonate

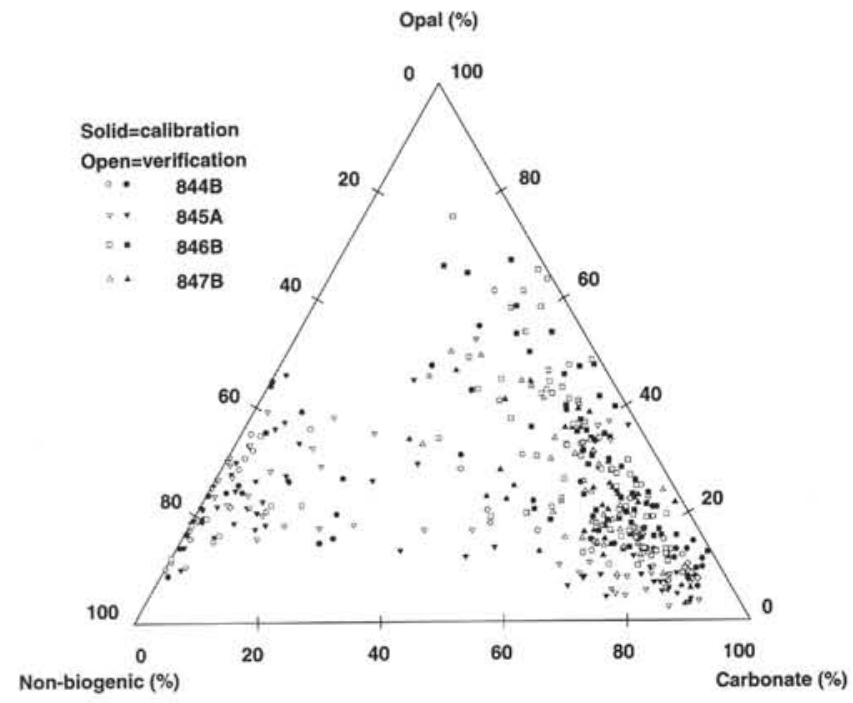

Figure 3. Ternary plot showing composition of calibration (solid symbols) and verification (open symbols) data sets. These ground-truth samples span a wide compositional range among the three end members considered here of calcium carbonate, opal, and nonbiogenic sediment.

mineral assemblage that dilutes the calcite. As long as the diluent reflectance spectrum is reasonably constant within the specific wavelength bands used in the calibration equation, this strategy will work well to predict the percentage of $\mathrm{CaCO}_{3}$. Of the five reflectance terms in the carbonate equation (at 95\% confidence interval), two are in the $\mathrm{nIR}(800-935 \mathrm{~nm})$ and three are in the middle visible bands (530-570 $\mathrm{nm}$ ). This is not surprising. Reflectance variations in the upper visible bands are variable, because they are dominated by the relative abundance of oxides (especially hematite and goethite; Barranco et al., 1989) and clays (especially smectite; Hunt and Salisbury, 1970; Hunt et al., 1971a). In terms of reflectance spectra, the diluent of $\mathrm{CaCO}_{3}$ is not constant in these bands. But outside of these bands, all of these minerals have relatively similar reflectance patterns, moderate from 800 to $950 \mathrm{~nm}$ and low at $<550 \mathrm{~nm}$. This explains why the predictive equation for calcite emphasizes the high and low ends of the spectrum, rather than the upper visible bands.

The prediction of $\mathrm{CaCO}_{3}$ concentration from GRAPE density follows Mayer (1991), but uses corrections of the raw GRAPE data for porosity rebound and compaction trends optimized for each site by Mayer et al. (this volume). Once these corrections were made, the percentage of $\mathrm{CaCO}_{3}$ was estimated by:

$$
\mathrm{CaCO}_{3} \%=-332.543 \rho^{2}+1112.673 \rho-835.525,
$$

where $\rho$ is the corrected GRAPE wet bulk density. For the same samples used for the calibration and verification of reflectance spectra, GRAPE predicted the percentage of $\mathrm{CaCO}_{3}$ with an RMS error of $\pm 13 \%\left(r^{2}=0.76\right)$ to $15 \%\left(r^{2}=0.80\right)$ (Figs. 4C and 4D). The GRAPE estimation here was less precise than that based on reflectance spectra, probably because of the presence of a relatively large and variable nonbiogenic component.

\section{Biogenic Opal}

Initial attempts to calibrate equations that estimate percentages of biogenic opal $\left(\mathrm{SiO}_{2} \cdot n \mathrm{H}_{2} \mathrm{O}\right)$ revealed that the reflectance spectra were sensitive to opal at low concentrations, but that calibration is less reliable at high concentrations of opal. To emphasize the lower concentrations more in the calibration equation, we performed the regression analysis after taking the log of opal concentration. As with the equation to estimate $\mathrm{CaCO}_{3}$ concentration, we allowed terms for 
Calibration
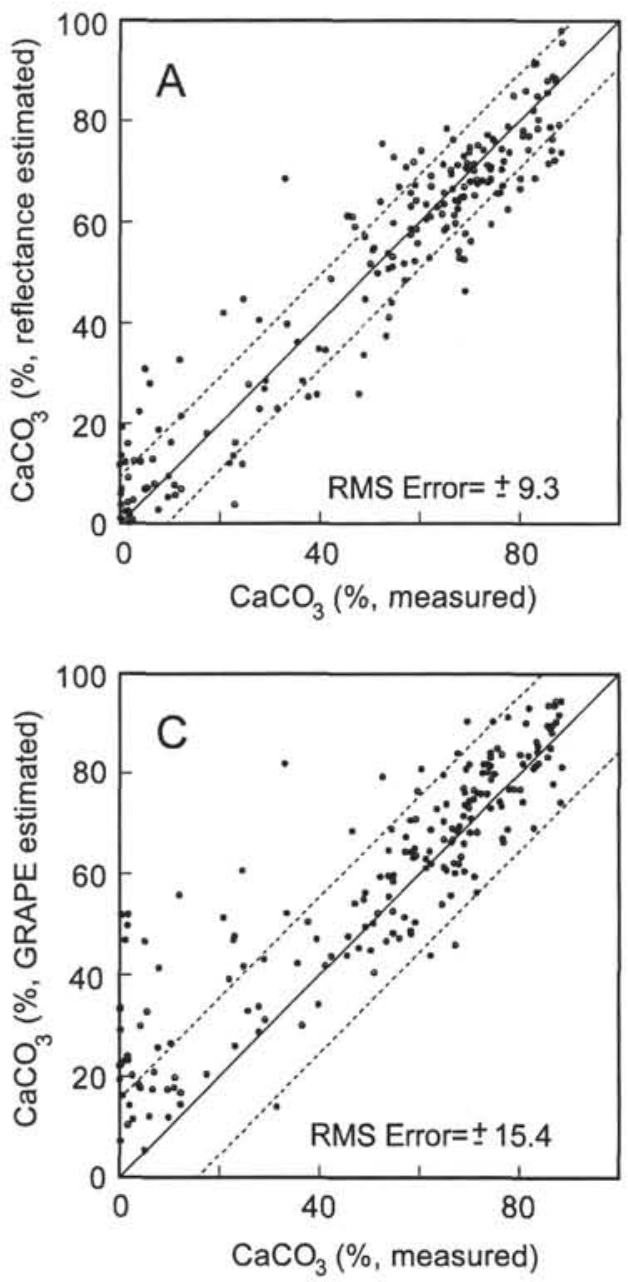

Verification
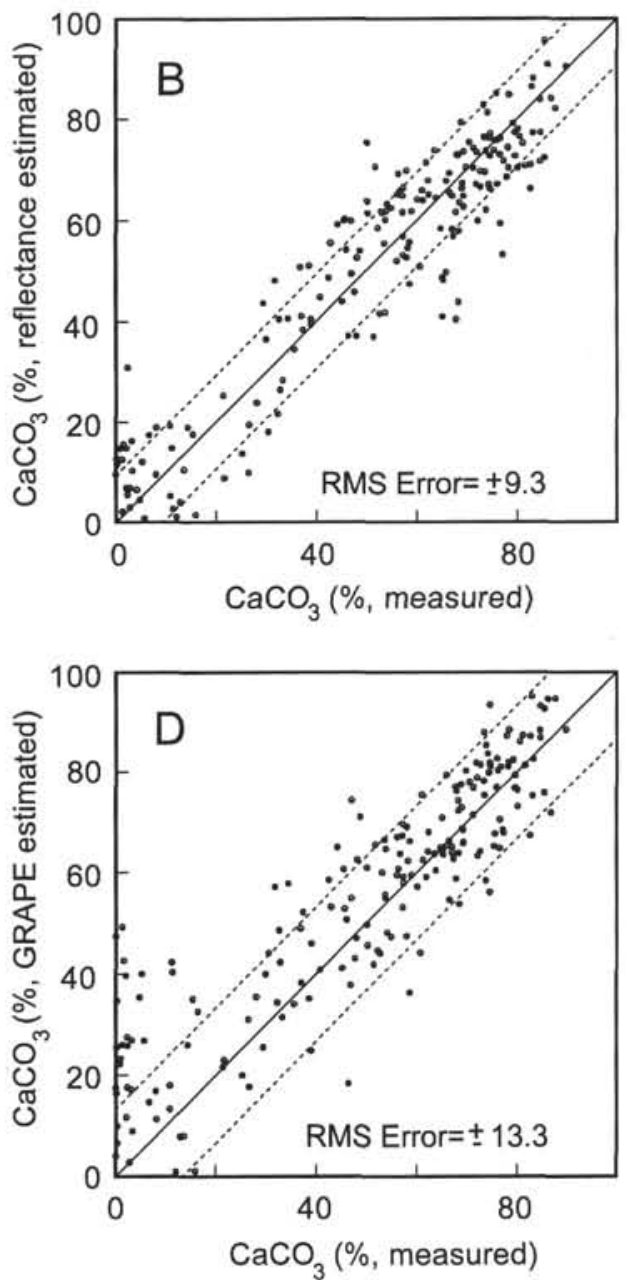

Figure 4. Estimated vs. measured percentages of $\mathrm{CaCO}_{3}$. A. Values estimated from reflectance, calibration data set (200 samples). B. Values estimated from reflectance, verification data set (198 samples). C. Values estimated from GRAPE density, calibration data set. D. Values estimated from GRAPE density, verification data set.

linear reflectance and the square of reflectance into the regression equation and kept only terms above $95 \%$ confidence. The resulting equation for predicting opal concentration is

$$
\begin{aligned}
& \log \left(\text { opal\% } \%=1.41 \times 10^{-3}\left(R_{550}\right)^{2}-1.82 \times 10^{-3}\left(R_{570}\right)^{2}\right. \\
& +9.86 \times 10^{-3}\left(R_{705}\right)+2.10 \times 10^{-4}\left(R_{935}\right)^{2}+1.25
\end{aligned}
$$

where $R$ is the average reflectance within a $20 \mathrm{~nm}$ bandwidth centered on the subscripted wavelength. After conversion to linear form, RMS errors for prediction of opal are $\pm 9.7 \%\left(r^{2}=0.44\right)$ for the calibration data set and $\pm 9.8 \%\left(r^{2}=0.52\right)$ for the verification data sets, respectively (Fig. 5). Although the percentage of variance in opal concentration accounted for by this equation $(44 \%-52 \%)$ is much lower than that of $\mathrm{CaCO}_{3}$, the estimates are significant at the 0.99 level $\left(r^{2}>r_{\text {(critical), }}^{2} 0.21\right.$ for $n=200$ ).

Of the four terms in this equation, two are in the middle visible bands ( $550-570 \mathrm{~nm})$, one is in the transition from red to $\mathrm{nIR}$ ( $705 \mathrm{~nm}$ ) and one is in the near infrared $(935 \mathrm{~nm})$. In these bands, reflectance of reagent opal is similar to that of calcite (Balsam, 1992). The regression equation thus is responding to the difference between the relatively high reflectance of opal vs. the combined spectral character of highly reflective carbonate and the less-reflective nonbiogenic sediments. This is not ideal, but would work as long as the other mineral associations remain constant.
The reflectance equation underestimates opal percentages at measured concentrations $>30 \%$, but the precision is reasonably good (RMS error $<6 \%$ ) for opal concentrations less than $40 \%$. Within the calibration data set, few samples with opal $>40 \%$ have nonbiogenic sediment $>30 \%$. Thus, anomalously low estimates of opal percentages at high concentrations suggest difficulties in separating opal from carbonate with the wavelengths measured here. Balsam (1992) noted that the spectra of pure calcite and opal diverge at wavelengths less than $500 \mathrm{~nm}$. Future refinements to the instrument that allow measurement of reflectance at lower visible and upper ultraviolet bands may improve the estimates of opal concentration. The present opal estimates, based on reflectance, do not capture the real amplitude of variability in the opal signal.

\section{Nonbiogenic Sediments}

Many common nonbiogenic sedimentary components are less reflective than either biogenic opal or calcium carbonate. Therefore, estimates of the concentration of nonbiogenic sediments from reflectance may be practical. Knowing the nonbiogenic component of sediments would also be useful for refining interpretation of GRAPE density data, for checking the estimates of the biogenic components, and for constraining causes of percentage variations in major lithologic components that may be diluted by nonbiogenic sediment sources. 
Table 1. Calibration data set.

\begin{tabular}{|c|c|c|c|c|c|c|c|c|c|c|}
\hline $\begin{array}{l}\text { Core, section, } \\
\text { interval }(\mathrm{cm})\end{array}$ & $\begin{array}{l}\text { Depth } \\
\text { (mbsf) }\end{array}$ & $\begin{array}{l}\text { Depth } \\
\text { (mcd) }\end{array}$ & $\begin{array}{l}\text { Depth } \\
\text { (rmcd) }\end{array}$ & $\begin{array}{c}\mathrm{CaCO}_{3} \\
\text { (\% measured) }\end{array}$ & $\begin{array}{c}\mathrm{CaCO}_{3} \\
\text { (\% estimated from } \\
\text { GRAPE density) }\end{array}$ & $\begin{array}{c}\mathrm{CaCO}_{3} \\
\text { (\% estimated from } \\
\text { reflectance) }\end{array}$ & $\begin{array}{c}\text { Opal } \\
\text { (\% measured) }\end{array}$ & $\begin{array}{c}\text { Opal } \\
\text { (\% estimated from } \\
\text { reflectance) }\end{array}$ & $\begin{array}{r}\text { Nonbiogenic } \\
\text { (\% measured) }\end{array}$ & $\begin{array}{c}\text { Nonbiogenic } \\
\text { (\% estimated from } \\
\text { reflectance) }\end{array}$ \\
\hline 138-844B- & & & & & & & & & & \\
\hline $1 \mathrm{H}-3,25-27$ & 3.26 & 3.26 & 3.26 & 5.60 & 32.81 & 7.31 & 24.10 & 25.94 & 70.29 & 65.85 \\
\hline $2 \mathrm{H}-2,46-47$ & 6.47 & 7.59 & 7.59 & 4.30 & 29.97 & -0.63 & 25.47 & 26.98 & 70.20 & 69.28 \\
\hline $2 \mathrm{H}-4,45-46$ & 9.46 & 10.59 & 10.59 & 23.00 & 47.71 & 4.00 & 19.91 & 27.42 & 57.08 & 63.04 \\
\hline $2 \mathrm{H}-6,31-33$ & 12.32 & 13.43 & 13.43 & 24.60 & 60.67 & 11.95 & 15.45 & 25.94 & 59.93 & 64.09 \\
\hline $3 \mathrm{H}-2,66-67$ & 16.17 & 18.41 & 18.41 & 1.70 & 49.80 & 4.43 & 18.79 & 26.79 & 79.50 & 69.52 \\
\hline $3 \mathrm{H}-3,66-67$ & 17.67 & 19.91 & 19.91 & 1.80 & 52.02 & 9.32 & 19.32 & 26.61 & 78.89 & 73.00 \\
\hline $3 \mathrm{H}-5,30-32$ & 20.31 & 22.55 & 22.55 & 0.40 & 51.94 & 13.69 & 16.41 & 26.61 & 83.21 & 61.11 \\
\hline $3 \mathrm{H}-7,30-32$ & 23.31 & 25.55 & 25.55 & 0.60 & 51.84 & 19.32 & 13.87 & 27.04 & 85.53 & 58.50 \\
\hline $4 \mathrm{H}-3,56-57$ & 27.07 & 18.53 & 18.53 & 0.30 & 29.14 & 3.95 & 23.60 & 25.88 & 76.07 & 71.61 \\
\hline $4 \mathrm{H}-5,56-57$ & 30.07 & 21.53 & 21.53 & 22.80 & 47.00 & 13.70 & 14.59 & 26.24 & 62.61 & 64.66 \\
\hline $4 \mathrm{H}-7,56-57$ & 33.07 & 24.53 & 24.53 & 1.20 & 46.90 & 12.38 & 8.67 & 24.66 & 90.13 & 69.38 \\
\hline $5 \mathrm{H}-2,65-66$ & 35.16 & 38.81 & 38.81 & 12.00 & 55.84 & 32.77 & 26.24 & 24.83 & 61.75 & 46.87 \\
\hline $5 \mathrm{H}-4,65-66$ & 38.16 & 41.81 & 41.81 & 20.80 & 51.35 & 42.05 & 26.55 & 28.77 & 52.65 & 28.29 \\
\hline $6 \mathrm{H}-3,67-68$ & 46.18 & 50.28 & 50.28 & 53.80 & 59.67 & 53.86 & 22.28 & 15.92 & 23.93 & 30.22 \\
\hline $6 \mathrm{H}-5,68-69$ & 49.19 & 53.28 & 53.28 & 7.90 & 41.46 & 18.82 & 39.08 & 22.34 & 53.00 & 62.23 \\
\hline $7 \mathrm{H}-2,70-71$ & 54.21 & 60.91 & 60.91 & 0.30 & 33.52 & 6.08 & 44.77 & 22.44 & 54.89 & 74.86 \\
\hline $7 \mathrm{H}-4,70-71$ & 57.21 & 63.91 & 63.91 & 0.40 & 22.23 & 7.08 & 43.95 & 23.28 & 55.67 & 72.57 \\
\hline $7 \mathrm{H}-6,70-71$ & 60.21 & 66.91 & 66.91 & 54.70 & 48.32 & 53.14 & 20.75 & 20.32 & 24.56 & 28.84 \\
\hline $8 \mathrm{H}-1,145-146$ & 62.96 & 70.09 & 70.09 & 37.70 & 50.53 & 25.32 & 30.90 & 24.27 & 31.39 & 49.55 \\
\hline $8 \mathrm{H}-4,30-32$ & 66.31 & 73.44 & 73.44 & 70.90 & 76.64 & 70.18 & 14.19 & 14.06 & 14.92 & 14.78 \\
\hline $8 \mathrm{H}-6,145-146$ & 70.46 & 77.61 & 77.61 & 28.90 & 43.17 & 26.98 & 54.95 & 29.44 & 16.19 & 41.34 \\
\hline $9 \mathrm{H}-2,66-67$ & 73.17 & 81.81 & 81.81 & 59.70 & 76.49 & 55.81 & 25.70 & 25.82 & 14.60 & 18.53 \\
\hline $9 \mathrm{H}-4,66-67$ & 76.17 & 84.81 & 84.81 & 73.60 & 81.86 & 71.24 & 16.29 & 14.76 & 10.11 & 7.11 \\
\hline $9 \mathrm{H}-6,66-67$ & 79.17 & 87.80 & 87.80 & 86.10 & 89.11 & 78.83 & 8.59 & 10.99 & 5.30 & 10.01 \\
\hline $10 \mathrm{H}-1,70-71$ & 81.21 & 91.84 & 91.84 & 87.00 & 93.56 & 72.25 & 8.13 & 15.31 & 4.87 & 9.99 \\
\hline $10 \mathrm{H}-3,70-71$ & 84.21 & 94.84 & 94.84 & 81.90 & 93.07 & 77.05 & 11.53 & 11.80 & 6.57 & 10.94 \\
\hline $10 \mathrm{H}-5,70-71$ & 87.21 & 97.84 & 97.84 & 86.40 & 88.13 & 74.22 & 9.62 & 14.66 & 3.98 & 7.73 \\
\hline $11 \mathrm{H}-1,70-71$ & 90.71 & 104.21 & 104.21 & 77.70 & 91.25 & 78.81 & 13.18 & 11.19 & 9.11 & 4.78 \\
\hline $11 \mathrm{H}-3,70-71$ & 93.71 & 107.21 & 107.21 & 73.60 & 80.25 & 67.87 & 21.09 & 23.39 & 5.29 & 7.28 \\
\hline $11 \mathrm{H}-5,70-71$ & 96.71 & 110.21 & 110.21 & 83.10 & 81.48 & 68.70 & 13.24 & 15.31 & 3.64 & 15.01 \\
\hline $11 \mathrm{H}-7,70-71$ & 99.71 & 113.21 & 113.21 & 74.30 & 78.78 & 59.62 & 21.09 & 32.28 & 4.59 & 12.70 \\
\hline $12 \mathrm{H}-2,65-66$ & 101.66 & 115.17 & 115.17 & 80.10 & 83.80 & 66.62 & 15.78 & 21.88 & 4.12 & 11.27 \\
\hline $12 \mathrm{H}-5,65-66$ & 106.16 & 119.68 & 119.68 & 86.50 & 89.60 & 76.49 & 11.38 & 14.49 & 2.11 & 1.29 \\
\hline $12 \mathrm{H}-7,65-66$ & 109.16 & 122.68 & 122.68 & 24.80 & 41.79 & 44.80 & 47.64 & 55.85 & 27.57 & 5.12 \\
\hline $15 \mathrm{H}-2,65-66$ & 130.16 & 145.15 & 145.15 & 81.30 & 90.10 & 85.92 & 13.24 & 8.04 & 5.44 & 6.89 \\
\hline $16 \mathrm{H}-4,100-102$ & 143.01 & 159.20 & 159.20 & 83.40 & 86.38 & 91.45 & 14.72 & 6.47 & 1.88 & 6.23 \\
\hline $18 \mathrm{H}-6,100-102$ & 165.01 & 182.07 & 182.07 & 87.30 & 94.44 & 87.79 & 9.86 & 8.18 & 2.84 & 11.48 \\
\hline $19 \mathrm{H}-6,100-102$ & 174.51 & 191.72 & 191.72 & 74.70 & 90.36 & 76.36 & 15.35 & 12.27 & 9.97 & 9.70 \\
\hline $20 \mathrm{H}-6,100-102$ & 184.01 & 202.65 & 202.65 & 88.30 & 94.46 & 73.82 & 6.27 & 15.28 & 5.44 & 11.65 \\
\hline $138-845 \mathrm{~A}-$ & & & & & & & & & & \\
\hline $1 \mathrm{H}-4,145-150$ & 5.98 & 5.96 & 5.96 & 3.00 & -56.98 & 12.64 & 24.10 & 32.36 & 72.93 & 54.33 \\
\hline $2 \mathrm{H}-1,40-42$ & 8.01 & 8.88 & 8.88 & 3.92 & 18.42 & 22.39 & 35.32 & 30.55 & 60.79 & 41.14 \\
\hline $2 \mathrm{H}-3,40-42$ & 11.01 & 11.90 & 11.90 & 6.00 & 12.20 & 28.04 & 37.24 & 26.85 & 56.74 & 40.66 \\
\hline $2 \mathrm{H}-5,40-42$ & 14.01 & 14.90 & 14.90 & 1.75 & 24.03 & 2.68 & 29.85 & 26.92 & 68.40 & 62.70 \\
\hline $3 \mathrm{H}-1,40-42$ & 17.51 & 18.36 & 18.36 & 5.08 & 5.57 & 30.92 & 35.97 & 28.91 & 58.95 & 32.04 \\
\hline $3 \mathrm{H}-3,40-42$ & 20.51 & 21.38 & 21.38 & 10.33 & 26.61 & 16.32 & 33.27 & 29.17 & 56.42 & 52.28 \\
\hline $3 \mathrm{H}-5,40-42$ & 23.51 & 24.38 & 24.38 & 4.25 & 17.75 & 12.47 & 24.49 & 26.48 & 71.24 & 55.90 \\
\hline $3 \mathrm{H}-7,40-42$ & 26.51 & 27.38 & 27.38 & 12.25 & 14.59 & 21.59 & 26.06 & 26.79 & 61.71 & 48.03 \\
\hline $4 \mathrm{H}-2,40-42$ & 28.51 & 30.65 & 30.65 & 6.67 & 17.54 & 13.01 & 26.30 & 26.30 & 67.01 & 56.73 \\
\hline $4 \mathrm{H}-4,40-42$ & 31.51 & 33.65 & 33.65 & 2.08 & 14.37 & 1.85 & 27.61 & 24.83 & 70.28 & 69.84 \\
\hline $4 \mathrm{H}-6,40-42$ & 34.51 & 36.65 & 36.65 & 2.67 & 20.26 & 0.84 & 9.89 & 25.47 & 87.44 & 69.11 \\
\hline $5 \mathrm{H}-1,100-102$ & 37.11 & 40.63 & 40.63 & 1.17 & 23.07 & 2.57 & 14.03 & 25.47 & 84.79 & 58.25 \\
\hline $5 \mathrm{H}-3,100-102$ & 40.11 & 43.63 & 43.63 & 5.08 & 46.65 & 7.00 & 15.92 & 26.36 & 79.01 & 60.82 \\
\hline $5 \mathrm{H}-6,102-104$ & 44.63 & 48.13 & 48.13 & 11.17 & 19.83 & 5.88 & 27.23 & 26.36 & 61.60 & 60.91 \\
\hline $6 \mathrm{H}-3,30-32$ & 48.91 & 52.98 & 52.98 & 1.75 & 10.50 & 16.06 & 29.79 & 27.54 & 68.43 & 55.96 \\
\hline $7 \mathrm{H}-3,99-101$ & 59.10 & 63.94 & 63.94 & 0.08 & 22.15 & 11.81 & 18.62 & 25.64 & 81.32 & 61.78 \\
\hline $10 \mathrm{H}-1,100-102$ & 84.61 & 93.10 & 93.10 & 9.83 & 12.03 & 9.64 & 20.32 & 24.27 & 69.84 & 69.13 \\
\hline $10 \mathrm{H}-3,103-105$ & 87.64 & 96.10 & 96.10 & 7.00 & 20.95 & 8.12 & 18.45 & 23.66 & 74.54 & 70.17 \\
\hline $10 \mathrm{H}-5,101-103$ & 90.62 & 99.10 & 99.10 & 11.00 & 17.86 & 7.86 & 17.34 & 23.44 & 71.66 & 69.71 \\
\hline $11 \mathrm{H}-1,100-102$ & 94.11 & 103.88 & 103.88 & 2.75 & 11.61 & 4.87 & 21.58 & 23.88 & 75.69 & 68.09 \\
\hline $11 \mathrm{H}-4,100-102$ & 98.61 & 108.38 & 108.38 & 9.67 & 17.52 & 5.49 & 22.49 & 24.10 & 67.86 & 66.56 \\
\hline $11 \mathrm{H}-6,100-102$ & 101.61 & 111.38 & 111.38 & 1.88 & 23.28 & 0.39 & 46.03 & 22.75 & 52.05 & 67.43 \\
\hline $12 \mathrm{H}-2,100-102$ & 105.11 & 116.45 & 116.45 & 23.17 & 26.00 & 16.24 & 44.98 & 26.00 & 31.88 & 55.35 \\
\hline $12 \mathrm{H}-4,100-102$ & 108.11 & 119.42 & 119.42 & 31.50 & 14.08 & 23.03 & 29.31 & 26.18 & 39.19 & 49.69 \\
\hline $12 \mathrm{H}-6,100-102$ & 111.11 & 122.45 & 122.45 & 7.75 & 25.75 & 2.98 & 21.33 & 23.66 & 70.93 & 64.06 \\
\hline $13 \mathrm{H}-2,100-102$ & 114.61 & 127.23 & 127.23 & 12.33 & 16.84 & 7.06 & 18.07 & 22.44 & 69.62 & 67.22 \\
\hline $13 \mathrm{H}-5,100-102$ & 119.11 & 131.73 & 131.73 & 0.25 & 7.34 & -1.67 & 26.67 & 22.28 & 73.09 & 73.89 \\
\hline $14 \mathrm{H}-2,100-102$ & 124.11 & 138.08 & 138.08 & 0.17 & 19.42 & -2.12 & 19.05 & 20.56 & 80.79 & 79.64 \\
\hline $14 \mathrm{H}-4,100-102$ & 127.11 & 141.10 & 141.10 & 0.67 & 16.30 & -2.78 & 21.09 & 21.23 & 78.22 & 76.68 \\
\hline $14 \mathrm{H}-6,100-102$ & 130.11 & 144.10 & 144.10 & 0.25 & 33.27 & 1.20 & 25.41 & 20.70 & 74.35 & 78.10 \\
\hline $15 \mathrm{H}-2,108-110$ & 133.69 & 149.56 & 149.56 & 0.17 & -3.17 & -6.69 & 21.43 & 20.65 & 78.41 & 81.14 \\
\hline $15 \mathrm{H}-5,108-110$ & 138.19 & 154.06 & 154.06 & 25.75 & 32.96 & 27.81 & 26.18 & 27.61 & 48.10 & 38.62 \\
\hline $16 \mathrm{H}-1,110-112$ & 141.71 & 159.44 & 159.44 & 36.59 & 30.11 & 28.54 & 13.24 & 36.73 & 50.15 & 47.38 \\
\hline $16 \mathrm{H}-3,110-112$ & 144.71 & 162.43 & 162.43 & 47.84 & 45.43 & 25.99 & 12.05 & 33.65 & 40.12 & 46.97 \\
\hline $16 \mathrm{H}-5,110-112$ & 147.71 & 165.43 & 165.43 & 51.67 & 52.26 & 49.93 & 13.80 & 19.32 & 34.53 & 32.91 \\
\hline $17 \mathrm{H}-1,101-103$ & 151.12 & 169.93 & 169.93 & 68.84 & 71.57 & 65.01 & 8.02 & 8.73 & 23.14 & 30.66 \\
\hline $17 \mathrm{H}-3,101-103$ & 154.12 & 172.93 & 172.93 & 74.34 & 73.12 & 70.85 & 4.52 & 5.74 & 21.14 & 25.46 \\
\hline $17 \mathrm{H}-5,101-103$ & 157.12 & 175.93 & 175.93 & 71.42 & 56.32 & 68.35 & 8.57 & 8.32 & 20.01 & 31.34 \\
\hline $18 \mathrm{H}-1,100-102$ & 160.61 & 179.87 & 179.87 & 68.92 & 66.10 & 66.94 & 8.30 & 8.65 & 22.78 & 26.91 \\
\hline $18 \mathrm{H}-4,100-102$ & 165.11 & 184.40 & 184.40 & 67.09 & 60.21 & 64.33 & 6.56 & 10.74 & 26.35 & 25.75 \\
\hline $18 \mathrm{H}-6,100-102$ & 168.11 & 187.40 & 187.40 & 80.76 & 74.38 & 78.11 & 7.14 & 8.34 & 12.10 & 14.38 \\
\hline $19 \mathrm{H}-2,100-102$ & 171.61 & 192.28 & 192.28 & 88.26 & 74.30 & 97.91 & 4.08 & 4.27 & 7.66 & -0.44 \\
\hline $19 \mathrm{H}-4,99-101$ & 174.60 & 195.24 & 195.24 & 88.42 & 81.29 & 95.55 & 3.40 & 5.51 & 8.18 & 1.70 \\
\hline $19 \mathrm{H}-6,100-102$ & 177.61 & 198.28 & 198.28 & 82.92 & 69.22 & 91.29 & 5.45 & 7.64 & 11.64 & 1.10 \\
\hline $20 \mathrm{H}-2,99-101$ & 181.10 & 203.41 & 203.41 & 75.01 & 79.86 & 76.03 & 12.05 & 10.00 & 12.95 & 12.94 \\
\hline $20 \mathrm{H}-4,101-103$ & 184.12 & 206.46 & 206.46 & 82.76 & 81.11 & 82.12 & 7.21 & 9.84 & 10.03 & 9.62 \\
\hline
\end{tabular}


Table 1 (continued).

\begin{tabular}{|c|c|c|c|c|c|c|c|c|c|c|}
\hline $\begin{array}{l}\text { Core, section, } \\
\text { interval }(\mathrm{cm})\end{array}$ & $\begin{array}{l}\text { Depth } \\
\text { (mbsf) }\end{array}$ & $\begin{array}{l}\text { Depth } \\
\text { (mcd) }\end{array}$ & $\begin{array}{l}\text { Depth } \\
\text { (rmcd) }\end{array}$ & $\begin{array}{c}\mathrm{CaCO}_{3} \\
\text { (\% measured) }\end{array}$ & $\begin{array}{c}\mathrm{CaCO}_{3} \\
\text { (\% estimated from } \\
\text { GRAPE density) }\end{array}$ & $\begin{array}{l}\mathrm{CaCO}_{3} \\
\text { (\% estimated from } \\
\text { reflectance) }\end{array}$ & $\begin{array}{c}\text { Opal } \\
\text { (\% measured) }\end{array}$ & $\begin{array}{l}\text { Opal } \\
\text { (\% estimated from } \\
\text { reflectance) }\end{array}$ & $\begin{array}{l}\text { Nonbiogenic } \\
(\% \text { measured) }\end{array}$ & $\begin{array}{l}\text { Nonbiogenic } \\
\text { (\% estimated from } \\
\text { reflectance) }\end{array}$ \\
\hline $20 \mathrm{H}-6,102-104$ & 187.13 & 209.45 & 209.45 & 81.76 & 83.49 & 74.34 & 7.14 & 11.07 & 11.10 & 17.01 \\
\hline $21 \mathrm{H}-2,100-102$ & 190.61 & 213.85 & 213.85 & 78.84 & 76.89 & 84.93 & 9.79 & 9.75 & 11.37 & 5.67 \\
\hline $21 \mathrm{H}-4,100-102$ & 193.61 & 216.85 & 216.85 & 78.01 & 76.82 & 73.74 & 8.57 & 13.03 & 13.42 & 16.11 \\
\hline $21 \mathrm{H}-6,100-102$ & 196.61 & 219.85 & 219.85 & 85.59 & 83.33 & 87.90 & 5.00 & 8.18 & 9.41 & 3.35 \\
\hline $22 \mathrm{H}-2,101-103$ & 200.12 & 225.35 & 225.35 & 83.67 & 85.50 & 78.48 & 6.10 & 10.33 & 10.24 & 15.43 \\
\hline $22 \mathrm{H}-4,100-102$ & 203.11 & 228.35 & 228.35 & 83.76 & 82.02 & 80.25 & 5.68 & 11.25 & 10.56 & 7.73 \\
\hline $22 \mathrm{H}-6,100-102$ & 206.11 & 231.35 & 231.35 & 83.59 & 84.32 & 84.91 & 8.81 & 10.91 & 7.60 & 0.89 \\
\hline 138-846B- & & & & & & & & & & \\
\hline 1H-4, 101-103 & 5.52 & 5.58 & 5.63 & 58.25 & 48.77 & 58.68 & 33.34 & 31.70 & 8.40 & 10.05 \\
\hline $2 \mathrm{H}-1,108-110$ & 8.09 & 8.84 & 8.94 & 62.17 & 43.91 & 61.09 & 36.31 & 31.77 & 1.55 & 11.43 \\
\hline $2 \mathrm{H}-3,97-99$ & 10.98 & 11.61 & 11.64 & 68.34 & 63.45 & 64.88 & 26.06 & 25.00 & 5.58 & 6.58 \\
\hline $2 \mathrm{H}-5,103-105$ & 14.04 & 14.63 & 14.64 & 77.67 & 68.87 & 62.47 & 15.78 & 31.84 & 6.55 & 7.10 \\
\hline $2 \mathrm{H}-7,51-53$ & 16.52 & 17.14 & 17.16 & 80.17 & 76.84 & 68.64 & 12.68 & $\begin{array}{l}1.07 \\
15.67\end{array}$ & 7.15 & 13.29 \\
\hline $3 \mathrm{H}-2,104-106$ & 19.05 & 21.33 & 21.55 & 69.17 & 73.74 & 57.77 & 23.55 & $\begin{array}{l}11.01 \\
31.33\end{array}$ & 7.30 & 11.49 \\
\hline $3 \mathrm{H}-4,104-106$ & 22.05 & 24.11 & 24.11 & 64.92 & 66.88 & 58.41 & 28.51 & 29.24 & 6.59 & 10.08 \\
\hline $4 \mathrm{H}-2,103-105$ & 28.54 & 31.67 & 31.67 & 50.75 & 50.29 & 54.44 & 39.17 & 35.16 & 10.05 & 10.76 \\
\hline $4 \mathrm{H}-4,103-105$ & 31.54 & 34.63 & 34.60 & 53.84 & 64.77 & 50.81 & 20.09 & 35.48 & 26.07 & 17.90 \\
\hline $4 \mathrm{H}-6,118-120$ & 34.69 & 37.85 & 37.88 & 71.59 & 68.33 & 67.66 & 23.71 & 25.47 & 4.68 & 7.63 \\
\hline $5 \mathrm{H}-2,104-106$ & 38.05 & 43.28 & 43.30 & 62.09 & 64.59 & 52.96 & 29.99 & 30.62 & 7.92 & 12.37 \\
\hline $5 \mathrm{H}-4,104-106$ & 41.05 & 46.12 & 45.97 & 57.17 & 64.51 & 48.48 & 31.62 & 31.41 & 11.18 & 21.69 \\
\hline $5 \mathrm{H}-6,104-106$ & 44.05 & 49.76 & 50.20 & 69.01 & 60.53 & 52.59 & 25.12 & 33.81 & 5.90 & 14.93 \\
\hline $6 \mathrm{H}-2,104-106$ & 47.55 & 54.06 & 54.06 & 50.25 & 44.94 & 51.78 & 39.99 & 30.76 & 9.73 & 16.25 \\
\hline $6 \mathrm{H}-4,104-106$ & 50.55 & 57.06 & 57.06 & 35.59 & 42.39 & 36.24 & 53.58 & 30.48 & 10.89 & 32.65 \\
\hline $6 \mathrm{H}-6,102-104$ & 53.53 & 60.02 & 60.02 & 39.42 & 47.12 & 25.82 & 50.12 & 38.99 & 10.50 & 27.28 \\
\hline $7 \mathrm{H}-2,104-106$ & 57.05 & 65.35 & 65.34 & 17.50 & 20.46 & 18.11 & 66.22 & 30.13 & 16.34 & 47.36 \\
\hline $7 \mathrm{H}-6,108-110$ & 63.09 & 71.67 & 71.94 & 27.92 & 28.79 & 22.95 & 67.14 & 39.54 & 4.93 & 29.74 \\
\hline $8 \mathrm{H}-1,38-40$ & 64.39 & 74.01 & 73.95 & 41.17 & 41.89 & 34.62 & 53.70 & 35.56 & 5.08 & 19.67 \\
\hline $8 \mathrm{H}-3,103-105$ & 68.04 & 77.72 & 77.72 & 22.00 & 39.24 & 12.22 & 64.86 & 34.99 & 13.16 & 34.05 \\
\hline $8 \mathrm{H}-5,103-105$ & 71.04 & 80.63 & 80.57 & 53.92 & 55.55 & 41.14 & 37.33 & 40.55 & 8.71 & 13.50 \\
\hline $9 \mathrm{H}-2,104-106$ & 76.05 & 85.86 & 85.50 & 54.50 & 69.01 & 44.09 & 35.16 & 34.43 & 10.31 & 19.06 \\
\hline $9 \mathrm{H}-4,104-106$ & 79.05 & 89.20 & 89.19 & 71.01 & 59.49 & 65.25 & 20.42 & 28.25 & 8.59 & 11.50 \\
\hline $9 \mathrm{H}-6,104-106$ & 82.05 & 92.47 & 92.65 & 76.67 & 67.08 & 67.20 & 16.98 & 22.39 & 6.33 & 1.72 \\
\hline $10 \mathrm{H}-2,114-116$ & 85.65 & 98.21 & 98.22 & 59.13 & 65.15 & 67.32 & 34.75 & 27.42 & 6.12 & 6.62 \\
\hline $10 \mathrm{H}-4,113-115$ & 88.64 & 101.23 & 101.32 & 48.84 & 55.00 & 33.78 & 47.32 & 51.52 & 3.80 & 9.17 \\
\hline $10 \mathrm{H}-6,103-105$ & 91.54 & 104.23 & 104.41 & 46.59 & 68.61 & 61.06 & 36.06 & 28.84 & 17.34 & 12.14 \\
\hline $11 \mathrm{H}-2,103-105$ & 95.04 & 108.84 & 109.05 & 67.67 & 68.52 & 64.06 & 22.75 & 23.28 & 9.58 & 10.69 \\
\hline $11 \mathrm{H}-4,100-102$ & 98.01 & 111.56 & 111.62 & 33.04 & 81.94 & 68.55 & 58.61 & 18.32 & 8.34 & 7.06 \\
\hline $11 \mathrm{H}-6,102-104$ & 101.03 & 114.81 & 115.03 & 51.00 & 40.56 & 54.91 & 47.64 & 45.29 & 1.37 & 7.11 \\
\hline $12 \mathrm{H}-4,103-105$ & 107.54 & 122.38 & 122.43 & 33.50 & 52.20 & 39.81 & 42.95 & 32.06 & 23.52 & 28.15 \\
\hline $12 \mathrm{H}-6,100-102$ & 110.51 & 125.74 & 126.11 & 58.25 & 64.46 & 63.15 & 33.50 & 29.11 & 8.23 & $\begin{array}{r}2.130 \\
7.69\end{array}$ \\
\hline $13 \mathrm{H}-2,103-105$ & 114.04 & 130.30 & 130.15 & 47.09 & 54.20 & 59.10 & 45.81 & 31.92 & 7.04 & 5.73 \\
\hline $13 \mathrm{H}-4,102-104$ & 117.03 & 133.65 & 133.95 & 55.92 & 47.30 & 67.00 & 35.32 & 29.24 & 8.75 & 9.98 \\
\hline $13 \mathrm{H}-6,102-104$ & 120.03 & 136.98 & 137.40 & 63.51 & 72.95 & 63.01 & 27.86 & 34.12 & 8.61 & 9.16 \\
\hline $14 \mathrm{H}-2,102-104$ & 123.53 & 140.70 & 140.76 & 73.59 & 75.96 & 77.19 & 13.58 & 12.62 & 12.84 & 7.25 \\
\hline $14 \mathrm{H}-4,102-104$ & 126.53 & 143.76 & 143.83 & 72.59 & 80.12 & 68.22 & 15.96 & 23.44 & 11.46 & 9.50 \\
\hline $14 \mathrm{H}-6,103-105$ & 129.54 & 146.75 & 146.82 & 76.51 & 83.77 & 74.43 & 13.34 & 18.32 & 10.16 & 2.01 \\
\hline $15 \mathrm{H}-3,102-104$ & 134.53 & 154.21 & 154.20 & 59.30 & 70.96 & 64.29 & 28.97 & 23.60 & 11.73 & 10.47 \\
\hline $15 \mathrm{H}-5,94-96$ & 137.45 & 157.32 & 157.48 & 57.34 & 67.42 & 70.88 & 33.96 & 20.84 & 8.73 & 13.22 \\
\hline $16 \mathrm{H}-1,104-106$ & 141.05 & 160.68 & 160.22 & 66.59 & 69.09 & 66.59 & 15.14 & 24.66 & 18.28 & 9.33 \\
\hline $16 \mathrm{H}-3,102-104$ & 144.03 & 164.24 & 164.25 & 58.25 & 70.73 & 65.79 & 18.79 & 20.46 & 22.95 & 13.34 \\
\hline $16 \mathrm{H}-6,102-104$ & 148.53 & 168.57 & 168.48 & 64.76 & 79.69 & 71.40 & 20.42 & 19.19 & 14.84 & 3.21 \\
\hline $17 \mathrm{H}-2,102-104$ & 152.03 & 173.25 & 173.22 & 64.84 & 61.36 & 63.59 & 19.32 & 30.97 & 15.85 & 4.52 \\
\hline $17 \mathrm{H}-4,102-104$ & 155.03 & 176.25 & 176.23 & 60.34 & 80.88 & 74.12 & 34.04 & 18.66 & 5.63 & 5.71 \\
\hline $17 \mathrm{H}-6,102-104$ & 158.03 & 179.14 & 178.99 & 69.26 & 80.82 & 70.67 & 18.97 & 22.70 & 11.77 & 9.94 \\
\hline $18 \mathrm{H}-2,103-105$ & 161.54 & 183.35 & 183.33 & 61.34 & 63.05 & 60.58 & 23.39 & 33.96 & 15.27 & 2.73 \\
\hline $18 \mathrm{H}-4,103-105$ & 164.54 & 186.36 & 186.35 & 74.26 & 83.17 & 68.33 & 17.06 & 25.06 & 8.67 & 10.78 \\
\hline $18 \mathrm{H}-6,102-104$ & 167.53 & 189.39 & 189.40 & 85.76 & 93.60 & 71.63 & 8.09 & 6.07 & 6.15 & 32.40 \\
\hline $19 \mathrm{H}-2,103-105$ & 171.04 & 194.82 & 194.82 & 72.76 & 81.84 & 74.24 & 13.24 & 16.94 & 14.01 & 4.39 \\
\hline $19 \mathrm{H}-4,103-105$ & 174.04 & 197.82 & 197.82 & 69.92 & 81.79 & 70.43 & 20.04 & 22.03 & 10.02 & 4.73 \\
\hline $19 \mathrm{H}-6,103-105$ & 177.04 & 200.73 & 200.64 & 74.26 & 84.11 & 68.53 & 19.23 & 25.06 & 6.50 & 7.40 \\
\hline $20 \mathrm{H}-3,103-105$ & 182.04 & 207.62 & 207.61 & 71.67 & 76.17 & 75.14 & 18.07 & 16.44 & 10.28 & 8.61 \\
\hline $20 \mathrm{H}-5,103-105$ & 185.04 & 211.23 & 211.46 & 67.59 & 83.98 & 71.24 & 18.62 & 22.03 & 13.81 & 5.31 \\
\hline $21 \mathrm{H}-1,103-105$ & 188.54 & 215.50 & 215.51 & 74.17 & 81.52 & 70.40 & 15.14 & 21.53 & 10.70 & 8.52 \\
\hline $21 \mathrm{H}-3,103-105$ & 191.54 & 218.47 & 218.47 & 72.34 & 75.97 & 68.15 & 21.63 & 27.29 & 6.04 & 5.36 \\
\hline $21 \mathrm{H}-5,103-105$ & 194.54 & 221.47 & 221.47 & 75.59 & 84.94 & 65.72 & 13.49 & 29.65 & 10.91 & -2.85 \\
\hline $22 \mathrm{H}-1,103-105$ & 198.04 & 228.47 & 228.46 & 69.51 & 90.39 & 71.70 & 20.37 & 18.79 & 10.12 & 4.70 \\
\hline $22 \mathrm{H}-3,103-105$ & 201.04 & 231.47 & 231.47 & 66.26 & 55.80 & 70.29 & 24.15 & 24.49 & 9.60 & -2.09 \\
\hline $22 \mathrm{H}-5,103-105$ & 204.04 & 234.46 & 234.45 & 54.84 & 59.86 & 72.86 & 41.69 & 19.63 & 3.44 & 5.44 \\
\hline 138-847B- & & & & & & & & & & \\
\hline $1 \mathrm{H}-1,124-126$ & 1.25 & 1.28 & 1.30 & 67.09 & 45.96 & 59.73 & 16.67 & 21.09 & 16.25 & 14.08 \\
\hline $1 \mathrm{H}-3,103-105$ & 4.04 & 4.02 & 4.02 & 58.34 & 48.22 & 57.50 & $\begin{array}{l}39.81 \\
39.81\end{array}$ & 24.04 & 1.88 & 16.68 \\
\hline $1 \mathrm{H}-5,19-21$ & 6.20 & 6.32 & 6.48 & 59.17 & 50.46 & 52.29 & 30.62 & 20.28 & 10.23 & 19.29 \\
\hline $2 \mathrm{H}-2,102-104$ & 9.03 & 9.16 & 9.17 & 67.59 & 68.55 & 62.61 & 17.62 & 22.65 & 14.80 & 11.65 \\
\hline $2 \mathrm{H}-4,102-104$ & 12.03 & 12.15 & 12.15 & 76.51 & 73.50 & 70.39 & 15.63 & 12.22 & 7.85 & 14.33 \\
\hline $2 \mathrm{H}-6,101-103$ & 15.02 & 15.07 & 14.99 & 86.67 & 77.94 & 88.80 & 12.74 & 7.45 & 0.58 & -0.68 \\
\hline $3 \mathrm{H}-2,102-104$ & 18.53 & 18.94 & 19.01 & 65.42 & 60.77 & 58.94 & 21.28 & 16.11 & 13.30 & 19.63 \\
\hline $3 \mathrm{H}-4,31-33$ & 20.82 & 21.12 & 21.09 & 45.71 & 47.65 & 61.22 & 23.23 & 21.09 & 31.07 & 13.51 \\
\hline $3 \mathrm{H}-6,102-104$ & 24.53 & 24.83 & 24.80 & 69.92 & 73.21 & 73.78 & 22.70 & 13.15 & 7.37 & 7.09 \\
\hline $4 \mathrm{H}-1,102-104$ & 26.53 & 29.35 & 29.35 & 76.84 & 66.29 & 71.99 & 21.93 & 14.79 & 1.23 & 10.07 \\
\hline $4 \mathrm{H}-3,102-104$ & 29.53 & 32.43 & 32.48 & 49.17 & 56.31 & 57.10 & 22.70 & 23.55 & 28.12 & 15.50 \\
\hline $4 \mathrm{H}-5,102-104$ & 32.53 & 35.45 & 35.56 & 49.17 & 49.58 & 44.78 & 25.18 & 19.28 & 25.67 & 30.66 \\
\hline $4 \mathrm{H}-7,32-34$ & 34.83 & 37.77 & 37.84 & 64.51 & 53.99 & 61.60 & 25.41 & 17.18 & 10.06 & 16.60 \\
\hline $5 \mathrm{H}-2,103-105$ & 37.54 & 40.32 & 40.32 & 66.92 & 62.20 & 61.38 & 23.44 & 21.93 & 9.65 & 13.76 \\
\hline $5 \mathrm{H}-4,103-105$ & 40.54 & 43.38 & 43.46 & 62.34 & 62.11 & 69.15 & 29.38 & 19.63 & 8.28 & 7.35 \\
\hline $5 \mathrm{H}-6,108-110$ & 43.59 & 46.34 & 46.27 & 57.00 & 51.49 & 51.87 & 31.77 & 26.79 & 11.24 & 18.86 \\
\hline $6 \mathrm{H}-1,102-104$ & 45.53 & 50.62 & 50.64 & 39.84 & 34.23 & 34.90 & 41.21 & 26.42 & 18.99 & 29.74 \\
\hline
\end{tabular}


Table 1 (continued).

\begin{tabular}{|c|c|c|c|c|c|c|c|c|c|c|}
\hline $\begin{array}{l}\text { Core, section, } \\
\text { interval }(\mathrm{cm})\end{array}$ & $\begin{array}{l}\text { Depth } \\
\text { (mbsf) }\end{array}$ & $\begin{array}{l}\text { Depth } \\
\text { (mcd) }\end{array}$ & $\begin{array}{l}\text { Depth } \\
\text { (rmcd) }\end{array}$ & $\begin{array}{c}\mathrm{CaCO}_{3} \\
\text { (\% measured) }\end{array}$ & $\begin{array}{c}\mathrm{CaCO}_{3} \\
\text { (\% estimated from } \\
\text { GRAPE density) }\end{array}$ & $\begin{array}{c}\mathrm{CaCO}_{3} \\
\text { (\% estimated from } \\
\text { reflectance) }\end{array}$ & $\begin{array}{c}\text { Opal } \\
\text { (\% measured) }\end{array}$ & $\begin{array}{c}\text { Opal } \\
\text { (\% estimated from } \\
\text { reflectance) }\end{array}$ & $\begin{array}{l}\text { Nonbiogenic } \\
\text { (\% measured) }\end{array}$ & $\begin{array}{c}\text { Nonbiogenic } \\
\text { (\% estimated from } \\
\text { reflectance) }\end{array}$ \\
\hline $6 \mathrm{H}-3,102-104$ & 48.53 & 53.47 & 53.33 & 53.34 & 46.70 & 37.52 & 35.81 & 25.06 & 10.82 & 32.48 \\
\hline $6 \mathrm{H}-5,103-105$ & 51.54 & 56.70 & 56.78 & 59.34 & 63.73 & 58.60 & 12.94 & 22.96 & 27.71 & 13.88 \\
\hline $6 \mathrm{H}-7,27-29$ & 53.78 & 59.01 & 59.17 & 29.17 & 31.22 & 28.50 & 46.67 & 37.33 & 24.15 & 28.95 \\
\hline $7 \mathrm{H}-2,103-105$ & 56.54 & 63.15 & 63.03 & 27.92 & 33.81 & 40.66 & 33.88 & 26.30 & 38.22 & 30.12 \\
\hline $7 \mathrm{H}-4,102-104$ & 59.53 & 66.12 & 65.99 & 45.50 & 43.91 & 61.21 & 28,12 & 23.23 & 26.38 & 11.50 \\
\hline $7 \mathrm{H}-6,103-105$ & 62.54 & 69.04 & 68.72 & 42.30 & 43.80 & 48.73 & 44.57 & 29.58 & 13.13 & 11.49 \\
\hline $8 \mathrm{H}-3,102-104$ & 67.53 & 75.92 & 76.06 & 64.84 & 70.52 & 65.85 & 16.52 & 18.75 & 18.64 & 9.10 \\
\hline $8 \mathrm{H}-5,100-102$ & 70.51 & 78.84 & 78.96 & 61.34 & 61.25 & 63.49 & 28.31 & 26.06 & 10.37 & 6.10 \\
\hline $9 \mathrm{H}-1,103-105$ & 74.04 & 83.38 & 83.22 & 66.67 & 66.84 & 76.25 & 20.61 & 15.24 & 12.73 & 4.18 \\
\hline $9 \mathrm{H}-3,102-104$ & 77.03 & 86.38 & 86.25 & 54.67 & 52.64 & 51.21 & 35.89 & 28.84 & 9.46 & 16.36 \\
\hline $9 \mathrm{H}-5,104-106$ & 80.05 & 89.47 & 89.42 & 80.67 & 81.97 & 77.11 & 11.94 & 13.21 & 7.40 & 3.51 \\
\hline $9 \mathrm{H}-7,33-35$ & 82.34 & 91.72 & 91.62 & 52.59 & 79.33 & 75.50 & 39.36 & 10.50 & 8.04 & 11.85 \\
\hline $10 \mathrm{H}-3,104-106$ & 86.55 & 96.80 & 96.81 & 70.92 & 74.90 & 71.42 & 21.78 & 20.23 & 7.29 & 3.13 \\
\hline $10 \mathrm{H}-5,23-25$ & 88.74 & 98.95 & 98.95 & 70.17 & 68.25 & 68.00 & 23.33 & 15.14 & 6.51 & 14.08 \\
\hline $10 \mathrm{H}-7,43-45$ & 91.94 & 102.15 & 102.15 & 54.75 & 58.59 & 59.83 & 38.73 & 21.63 & 6.56 & 11.33 \\
\hline $11 \mathrm{H}-2,33-35$ & 93.84 & 106.60 & 106.40 & 66.34 & 66.32 & 69.51 & 16.67 & 17.30 & 17.00 & 5.15 \\
\hline $11 \mathrm{H}-4,33-35$ & 96.84 & 109.70 & 109.66 & 69.17 & 76.18 & 46.28 & 16.56 & 22.75 & 14.27 & 22.69 \\
\hline $11 \mathrm{H}-6,33-35$ & 99.84 & 112.68 & 112.63 & 70.09 & 74.60 & 74.78 & 12.08 & 15.14 & 17.84 & 2.14 \\
\hline $12 \mathrm{H}-2,33-35$ & 103.34 & 116.39 & 116.41 & 86.17 & 85.05 & 78.78 & 7.62 & 10.40 & 6.21 & 3.28 \\
\hline $12 \mathrm{H}-4,33-35$ & 106.34 & 119.44 & 119.50 & 68.01 & 69.54 & 52.92 & 14.13 & 16.07 & 17.85 & 28.07 \\
\hline $12 \mathrm{H}-6,33-35$ & 109.34 & 122.35 & 122.33 & 76.17 & 74.38 & 65.81 & 13.00 & 13.55 & 10.84 & 18.35 \\
\hline $13 \mathrm{H}-1,82-84$ & 111.83 & 125.97 & 126.09 & 67.90 & 62.03 & 54.25 & 18.49 & 17.46 & 13.60 & 20.70 \\
\hline $13 \mathrm{H}-3,41-43$ & 114.42 & 128.41 & 128.40 & 70.34 & 71.04 & 56.21 & 14.13 & 14.59 & 15.55 & 26.23 \\
\hline $13 \mathrm{H}-5,33-35$ & 117.34 & 131.36 & 131.39 & 85.59 & 89.58 & 85.57 & 6.38 & 6.97 & 8.02 & 4.37 \\
\hline $13 \mathrm{H}-7,33-35$ & 120.34 & 134.40 & 134.46 & 87.88 & 91.71 & 79.23 & 3.06 & 8.85 & 9.06 & 6.81 \\
\hline $14 \mathrm{H}-2,33-35$ & 122.34 & 137.88 & 137.97 & 87.26 & 90.30 & 88.35 & 5.98 & 6.03 & 6.76 & 0.36 \\
\hline $14 \mathrm{H}-4,102-104$ & 126.03 & 141.52 & 141.53 & 68.84 & 77.02 & 73.06 & 15.14 & 13.96 & 16.03 & 10.35 \\
\hline $14 \mathrm{H}-6,102-104$ & 129.03 & 144.54 & 144.59 & 65.42 & 73.93 & 78.43 & 24.60 & 16.67 & 10.00 & 2.54 \\
\hline $15 \mathrm{H}-1,64-66$ & 130.65 & 145.75 & 145.47 & 52.17 & 59.49 & 64.03 & 35.89 & 22.13 & 11.93 & 9.35 \\
\hline $15 \mathrm{H}-3,33-35$ & 133.34 & 146.65 & 145.78 & 62.34 & 68.86 & 67.08 & 24.60 & 23.12 & 13.08 & 6.91 \\
\hline $15 \mathrm{H}-6,33-35$ & 137.84 & 148.16 & 146.28 & 58.84 & 63.24 & 71.83 & 31.41 & 17.95 & 9.74 & 7.54 \\
\hline
\end{tabular}
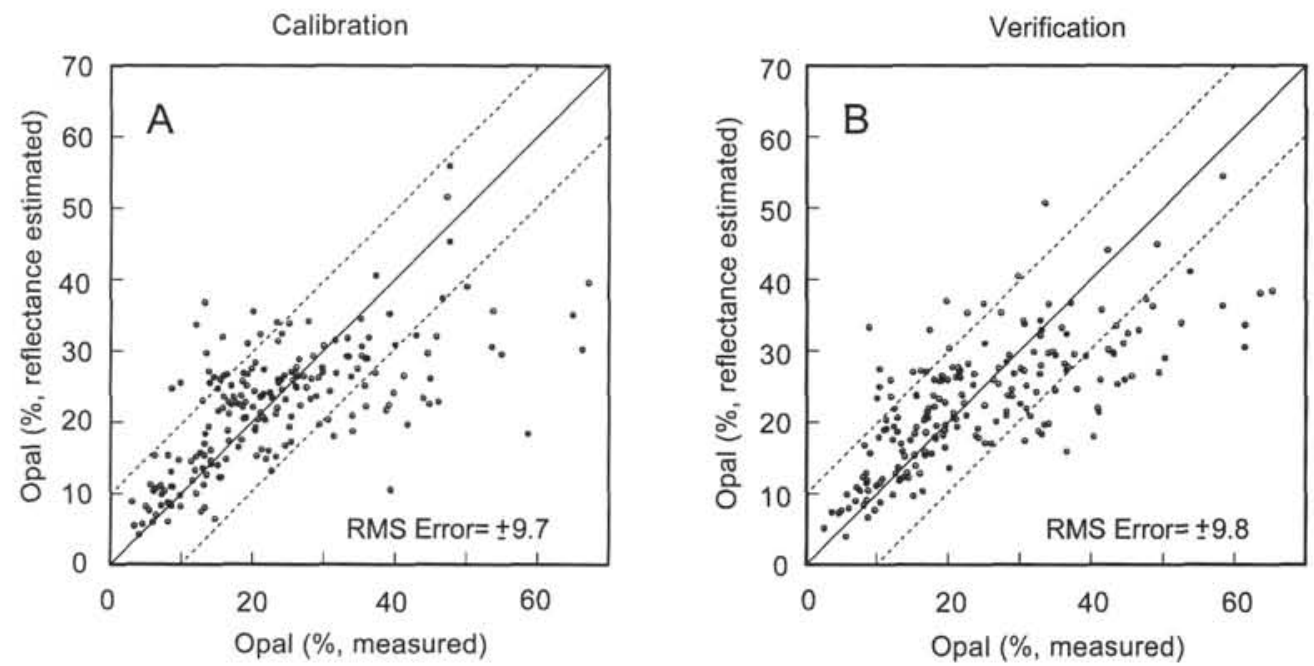

Figure 5. Estimated vs. measured percentages of biogenic opal. A. Values estimated from reflectance, calibration data set. B. Values estimated from reflectance, verification data set. The reflectance equation tends to underestimate opal percentages at higher concentrations.

Nonbiogenic material can include various components, such as clay minerals, oxides, sulfides, and sulfates, and with our simplistic method of calculation also includes traces of organic matter. We proceeded because the nonbiogenic sediments in this area are relatively simple, composed almost entirely of smectite clay (Heath et al., 1974; Schumann and Nagel, 1982; Zimmerman, 1982), although minor or trace quantities of barite, kaolinite, illite, quartz, and zeolites may be present.

Calibration of estimates for nonbiogenic sediment is shown in Figure 6. The nonbiogenic component ranges from $0 \%$ to $90 \%$ in the calibration data set. The regression equation again allows for linear and squared terms of reflectance and keeps only the terms above the $95 \%$ confidence interval. Concentration of total nonbiogenic sediments can be estimated with

$$
\begin{gathered}
\text { Nonbiogenic\% }=-3.94\left(R_{470}\right)+1.83 \times 10^{2}\left(R_{470}\right)^{2}+22.6\left(R_{665}\right) \\
-2.44 \times 10^{-1}\left(R_{685}\right)^{2}-25.2\left(R_{705}\right)+4.83 \times 10^{-2}\left(R_{705}\right)^{2}+1.51\left(R_{935}\right) \\
+138.8
\end{gathered}
$$

where $R$ is the average reflectance within a $20-\mathrm{nm}$ bandwidth centered on the subscripted wavelength. RMS error on predictions of nonbiogenic sediment is $\pm 10.2 \%\left(r^{2}=0.84\right)$ for the calibration data set, and $\pm 10.4 \%\left(r^{2}=0.83\right)$ for the verification data set. This indicates that the equation reproduces at least $83 \%$ of the variance in the ground-truth data set. Although the measured points are clustered at nonbiogenic concentrations of less than $20 \%$, the scatter of estimates appears relatively constant over the full range of variation. Three of the five different wavelength bands that appear as terms in this equation do not appear in the equations to predict biogenic calcium carbonate or 
Calibration

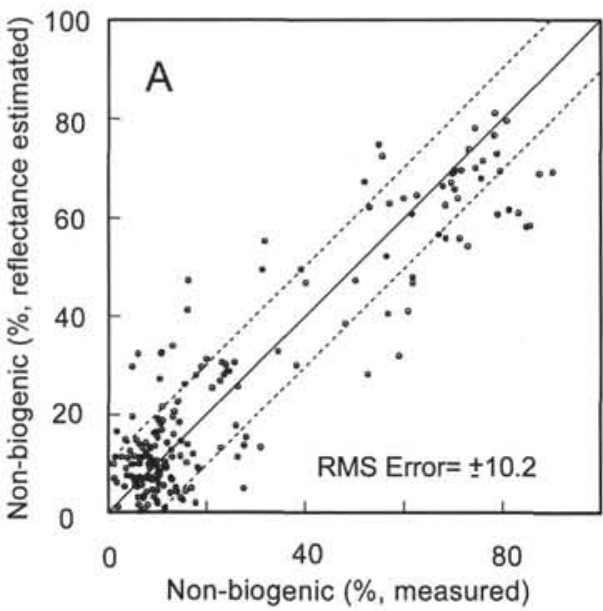

Verification

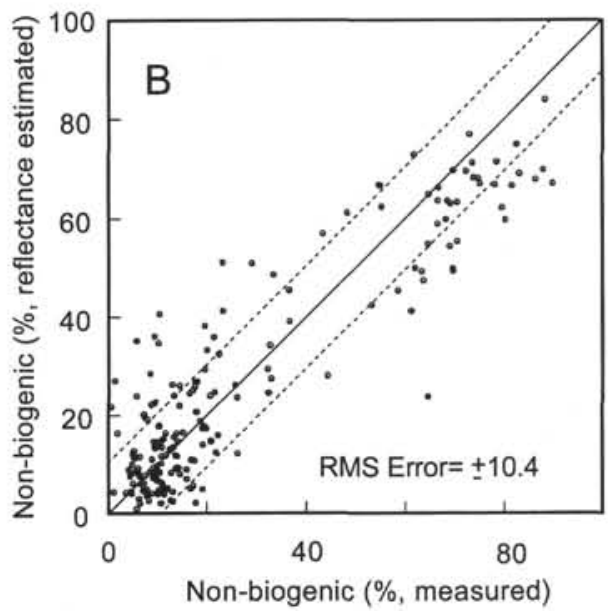

Figure 6. Estimated vs. measured percentages of nonbiogenic sediments. Measured values approximate the percentage of nonbiogenic sediment as $100-\left(\mathrm{CaCO}_{3} \%+\right.$ opal\%) without attempting to isolate the various components contributing to the material. The major contributor to nonbiogenic sediment is smectite clay. A. Values estimated from reflectance, calibration data set. B. Values estimated from reflectance, verification data set.

biogenic opal. This suggests some independence of the estimates of nonbiogenic and biogenic components. Carbonate and nonbiogenic sediments are inversely correlated in the calibration and verification data sets, however, so we cannot confirm that these two parameters can be estimated independently of each other.

\section{DOWNHOLE ESTIMATES}

\section{Eastern Pacific Sediment History}

Here, we estimate time series of sedimentary percentages of $\mathrm{CaCO}_{3}$, opal, and nonbiogenic sediment in Site 846 from the eastern transect of Leg 138, southwest of the Galapagos Archipelago. Our purpose is to evaluate the potential for multicomponent reconstructions of lithologic history from reflectance spectroscopy. The time scale we used is based on benthic foraminifer oxygen isotope stratigraphy in Holes $846 \mathrm{~B}, 846 \mathrm{C}$, and $846 \mathrm{D}(0-1.8 \mathrm{Ma}$ by Mix et al., this volume; $1.8-3.6 \mathrm{Ma}$ by Shackleton et al., this volume).

Time series of the three components estimated from reflectance are given in Figure 7. In this figure, ground-truth analyses are shown as solid dots. The two estimated components that vary most are $\mathrm{CaCO}_{3}$ and nonbiogenic sediments, and these are strongly negatively correlated $(r=-0.95)$, as they were in the calibration and verification data sets. The estimates of $\mathrm{CaCO}_{3}$ appear reasonable throughout the past 3.6 Ma (Fig. 7A).

Estimated opal is less variable than the other components. This low amplitude is partially an artifact of the failure of the regression equation to estimate high opal concentrations. This is shown in Figure $7 \mathrm{~B}$, where discrete opal measurements (filled circles) often are higher than downhole opal estimated from reflectance spectra.

The estimates of nonbiogenic sediment percentages are somewhat more reliable that those of opal, but at times they overestimate the true concentration of nonbiogenic sediments, such as in the interval between 1.5 and $2.1 \mathrm{Ma}$ (Fig. 7B). The intervals of major disagreement between reflectance estimates and ground-truth measurements of nonbiogenic sediments may indicate the presence of a nonbiogenic lithologic component that is not well represented in the calibration data set. Our partially successful attempt to predict nonbiogenic sediment composition downhole focuses our attention on areas where more study will be needed to define the lithologic character. Our estimate of nonbiogenic sediment concentration in the ground-truth data set was simplistic and, clearly, can be improved.
We conclude that the estimates of $\mathrm{CaCO}_{3}$ percentage from reflectance spectroscopy are useable for studying geologic history in these sites, that the estimates of nonbiogenic sediment are useful but require additional study to better characterize its many components, and that estimates of opal are not yet reliable in all situations.

\section{Comparing GRAPE with Reflectance Estimates of $\mathrm{CaCO}_{3}$ Percentages}

In Figure 8, we compare downhole estimates of $\mathrm{CaCO}_{3}$ based on reflectance and GRAPE density for typical 50-m intervals of APC and XCB cores from Site 846. In APC cores (Fig. 8A), clearly, both estimates are sensing the same carbonate variations, although in detail the records sometimes diverge. Estimation of $\mathrm{CaCO}_{3}$ percentages by both GRAPE and reflectance correspond reasonably well to downhole discrete carbonate measurements (denoted by filled circles).

GRAPE measurements may be influenced strongly by drilling disturbance, such as injection of water (common in the upper portion of Section 1 in many APC cores), or the tendency of sediments recovered by XCB to break into "biscuits" surrounded by soupy disturbed sediments near the core edges. Because GRAPE measurements integrate the complete cross section of the core, they cannot avoid the disturbed intervals, which appear as anomalously low density (and thus low inferred $\mathrm{CaCO}_{3}$ ) values. This biscuit effect is shown in estimates of downhole carbonate percentage for XCB cores from depths of 280 to $330 \mathrm{rmcd}$ in Hole 846B (Fig. 8B). Here, the GRAPE estimates of $\mathrm{CaCO}_{3}$ exhibit occasional spikes of anomalously low percentage. Reflectance is less sensitive to the biscuit effect in the XCB sections, because sediment biscuits tend to meet with minimal gaps between them where reflectance is measured, along the axis of the core. We conclude that reflectance estimates are less sensitive to errors associated with sediment disturbance and biscuit formation induced by XCB coring in partially lithified sediments.

However, reflectance may be sensitive to other errors in $\mathrm{XCB}$ cores. For example, XCB cores are sometimes difficult to split, and surface roughness associated with imperfect splitting may induce errors in our measurements of reflectance spectra. We tested for this effect by estimating $\mathrm{CaCO}_{3}$ percentage by GRAPE and by reflectance for 107 samples from XCB cores that have chemical ground-truth measurements at the same location as both GRAPE and reflectance measurements (Table 3 ). In these samples, which were carefully 
Table 2. Verification data set.

\begin{tabular}{|c|c|c|c|c|c|c|c|c|c|c|}
\hline $\begin{array}{l}\text { Core, section, } \\
\text { interval }(\mathrm{cm})\end{array}$ & $\begin{array}{l}\text { Depth } \\
\text { (mbsf) }\end{array}$ & $\begin{array}{l}\text { Depth } \\
\text { (mcd) }\end{array}$ & $\begin{array}{l}\text { Depth } \\
\text { (rmcd) }\end{array}$ & $\begin{array}{c}\mathrm{CaCO}_{3} \\
\text { (\% measured) }\end{array}$ & $\begin{array}{c}\mathrm{CaCO}_{3} \\
\text { (\% estimated from } \\
\text { GRAPE density) }\end{array}$ & $\begin{array}{c}\mathrm{CaCO}_{3} \\
\text { (\% estimated from } \\
\text { reflectance) }\end{array}$ & $\begin{array}{c}\text { Opal } \\
\text { (\% measured) }\end{array}$ & $\begin{array}{c}\text { Opal } \\
\text { (\% estimated from } \\
\text { reflectance) }\end{array}$ & $\begin{array}{l}\text { Nonbiogenic } \\
(\% \text { measured) }\end{array}$ & $\begin{array}{l}\text { Nonbiogenic } \\
\text { (\% estimated from } \\
\text { reflectance) }\end{array}$ \\
\hline 138-844B- & & & & & & & & & & \\
\hline $2 \mathrm{H}-1,31-33$ & 4.82 & 5.93 & 5.93 & 4.90 & 35.42 & 4.65 & 21.49 & 26.87 & 73.61 & 71.22 \\
\hline $2 \mathrm{H}-3,27-29$ & 7.78 & 8.89 & 8.89 & 16.50 & 32.64 & -4.88 & 21.66 & 26.20 & 61.84 & 72.85 \\
\hline $2 \mathrm{H}-5,45-46$ & 10.96 & 12.09 & 12.09 & 11.50 & 40.35 & 2.84 & 21.69 & 27.21 & 66.81 & 66.32 \\
\hline $2 \mathrm{H}-7,25-26$ & 13.76 & 14.89 & 14.89 & 5.80 & 26.89 & 0.98 & 16.12 & 27.12 & 78.08 & 66.87 \\
\hline $3 \mathrm{H}-3,30-32$ & 17.31 & 19.55 & 19.55 & 0.10 & 47.44 & 9.77 & 11.97 & 25.86 & 87.93 & 69.85 \\
\hline $3 \mathrm{H}-4,36-38$ & 18.87 & 21.13 & 21.13 & 5.40 & 40.06 & 12.17 & 15.06 & 26.96 & 79.54 & 62.21 \\
\hline $3 \mathrm{H}-6,30-32$ & 21.81 & 24.05 & 24.05 & 0.40 & 34.77 & 11.73 & 18.05 & 25.90 & 81.55 & 66.67 \\
\hline $4 \mathrm{H}-2,56-57$ & 25.57 & 17.03 & 17.03 & 11.20 & 42.32 & 14.90 & 20.57 & 27.63 & 68.23 & 59.85 \\
\hline $4 \mathrm{H}-4,56-57$ & 28.57 & 20.03 & 20.03 & 2.20 & 39.70 & 7.03 & 19.31 & 25.95 & 78.49 & 71.44 \\
\hline $4 \mathrm{H}-6,56-57$ & 31.57 & 23.03 & 23.03 & 1.40 & 49.29 & 12.62 & 15.54 & 23.72 & 83.06 & 69.08 \\
\hline $5 \mathrm{H}-1,65-66$ & 33.66 & 37.31 & 37.31 & 47.10 & 74.35 & 49.46 & 20.67 & 21.25 & 32.23 & 29.46 \\
\hline $5 \mathrm{H}-3,65-66$ & 36.66 & 40.31 & 40.31 & 48.80 & 71.04 & 53.95 & 18.26 & 17.65 & 32.94 & 27.52 \\
\hline $5 \mathrm{H}-5,119-120$ & 40.20 & 43.83 & 43.83 & 53.80 & 55.68 & 60.09 & 36.55 & 15.88 & 9.65 & 22.69 \\
\hline $6 \mathrm{H}-2,78-79$ & 44.79 & 48.87 & 48.87 & 57.20 & 67.36 & 61.60 & 16.73 & 15.84 & 26.07 & 23.68 \\
\hline $6 \mathrm{H}-4,68-69$ & 47.69 & $\begin{array}{l}40.01 \\
51.78\end{array}$ & 51.78 & 67.80 & 77.04 & 61.61 & 12.99 & 13.64 & 19.21 & 14.22 \\
\hline $6 \mathrm{H}-6,68-69$ & 50.69 & 54.78 & 54.78 & 32.60 & 48.64 & 40.45 & 34.03 & 19.74 & 33.37 & 48.61 \\
\hline $7 \mathrm{H}-1,70-71$ & 52.71 & 59.40 & 59.40 & 10.90 & 13.44 & 19.32 & 35.84 & 33.21 & 53.26 & 42.42 \\
\hline $7 \mathrm{H}-5,70-71$ & 58.71 & 65.41 & 65.41 & 39.00 & 46.01 & 39.50 & 28.29 & 23.65 & 32.71 & 34.28 \\
\hline $7 \mathrm{H}-7,70-71$ & 61.71 & 68.41 & 68.41 & 57.40 & 59.20 & 53.10 & 21.05 & 20.39 & 21.55 & 24.74 \\
\hline $8 \mathrm{H}-5,145-146$ & 68.96 & 76.11 & 76.11 & 69.30 & 68.51 & 62.71 & 22.51 & 20.93 & 8.19 & 19.15 \\
\hline $9 \mathrm{H}-1,66-67$ & 71.67 & 80.32 & 80.32 & 28.10 & 35.50 & 23.86 & 61.48 & 33.56 & 10.43 & 40.67 \\
\hline $9 \mathrm{H}-3,66-67$ & 74.67 & 83.31 & 83.31 & 47.70 & 43.02 & $\begin{array}{l}25.00 \\
45.79\end{array}$ & 30.91 & 21.84 & 21.39 & 35.93 \\
\hline $9 \mathrm{H}-5,66-67$ & 77.67 & 86.30 & 86.30 & 73.80 & 85.31 & 73.74 & 15.27 & 15.33 & 10.93 & 6.43 \\
\hline $9 \mathrm{H}-7,32-34$ & 80.33 & 88.97 & 88.97 & 87.60 & 94.62 & 82.15 & 7.68 & 10.47 & 4.72 & 4.67 \\
\hline $10 \mathrm{H}-2,70-71$ & 82.71 & 93.34 & 93.34 & 85.40 & 92.56 & 72.38 & 9.08 & 15.64 & 5.52 & 11.44 \\
\hline $11 \mathrm{H}-2,70-71$ & 92.21 & 105.71 & 105.71 & 82.60 & 87.12 & 71.05 & 8.30 & 16.75 & 9.10 & 8.04 \\
\hline $11 \mathrm{H}-4,70-71$ & 95.21 & 108.71 & 108.71 & 69.80 & 80.22 & 70.49 & 25.09 & 17.06 & 5.11 & 12.38 \\
\hline $11 \mathrm{H}-6,70-71$ & 98.21 & 111.71 & 111.71 & 80.50 & 86.04 & 76.49 & 13.19 & 11.81 & 6.31 & 3.21 \\
\hline $12 \mathrm{H}-1,65-66$ & 100.16 & 113.67 & 113.67 & 69.30 & 65.88 & 64.83 & 21.08 & 26.25 & 9.62 & 8.30 \\
\hline $12 \mathrm{H}-6,65-66$ & 107.66 & 121.18 & 121.18 & 56.80 & 63.80 & 65.92 & 26.32 & 25.85 & 16.88 & 11.16 \\
\hline $13 \mathrm{H}-4,100-102$ & 114.51 & 128.31 & 128.31 & 84.60 & 86.80 & 77.32 & 10.44 & 11.48 & 4.96 & 10.17 \\
\hline $15 \mathrm{H}-5,0-5$ & 134.03 & 149.02 & 149.02 & 78.40 & 88.40 & 84.77 & 15.06 & 9.70 & 6.54 & 7.61 \\
\hline $18 \mathrm{H}-5,0-5$ & 162.53 & 179.57 & 179.57 & 82.90 & 95.07 & 86.41 & 12.21 & 9.90 & 4.89 & 8.30 \\
\hline $19 \mathrm{H}-2,100-102$ & 168.51 & 185.72 & 185.72 & 86.10 & 94.53 & 90.81 & 8.74 & 6.71 & 5.16 & 6.29 \\
\hline $20 \mathrm{H}-2,100-102$ & 178.01 & 196.65 & 196.65 & 74.60 & 93.36 & 72.73 & 19.24 & 18.16 & 6.16 & 9.40 \\
\hline $138-845 \mathrm{~A}-$ & & & & & & & & & & \\
\hline $1 \mathrm{H}-3,40-42$ & 3.41 & 3.42 & 3.42 & 2.92 & 2.98 & 7.02 & 30.46 & 34.14 & 66.62 & 63.65 \\
\hline $1 \mathrm{H}-5,22-24$ & 6.23 & 6.22 & 6.22 & 2.92 & -14.26 & 3.15 & 28.49 & 29.30 & 68.59 & 63.64 \\
\hline $2 \mathrm{H}-2,40-42$ & 9.51 & 10.40 & 10.40 & 3.42 & 9.13 & 10.43 & 31.96 & 29.76 & 64.62 & 54.85 \\
\hline $2 \mathrm{H}-4,40-42$ & 12.51 & 13.37 & $\begin{array}{l}10.40 \\
13.37\end{array}$ & 3.33 & 26.94 & 16.31 & 34.65 & 29.81 & 62.02 & 49.94 \\
\hline $2 \mathrm{H}-6,101-103$ & 16.12 & 17.00 & 17.00 & 1.67 & 42.66 & 15.61 & 34.99 & 29.38 & 63.34 & 49.30 \\
\hline $3 \mathrm{H}-2,40-42$ & 19.01 & 19.88 & 19.88 & 2.25 & 11.84 & 14.89 & 39.20 & 29.21 & 58.55 & 45.37 \\
\hline $3 \mathrm{H}-4,40-42$ & 22.01 & 22.88 & 22.88 & 6.75 & 14.79 & 17.50 & 23.63 & 26.70 & 69.62 & 49.91 \\
\hline $3 \mathrm{H}-6,40-42$ & 25.01 & 25.88 & 25.88 & 2.42 & 17.69 & 5.65 & 32.81 & 26.70 & 64.77 & 64.93 \\
\hline $4 \mathrm{H}-1,40-42$ & 27.01 & 29.15 & 29.15 & 8.17 & 11.48 & 19.02 & 28.12 & 28.37 & 63.71 & 47.45 \\
\hline $4 \mathrm{H}-3,40-42$ & 30.01 & 32.15 & 32.15 & $\begin{array}{l}0.17 \\
1.08\end{array}$ & 23.36 & 14.82 & 29.24 & 26.76 & 69.68 & 49.41 \\
\hline $4 \mathrm{H}-5,101-103$ & 33.62 & 35.75 & 35.75 & 1.42 & 26.07 & 2.31 & 23.49 & 25.19 & 75.09 & 67.07 \\
\hline $4 \mathrm{H}-7,40-42$ & 36.01 & 38.15 & 38.15 & 3.25 & 17.21 & -0.21 & 10.40 & 24.89 & 86.35 & 67.90 \\
\hline $5 \mathrm{H}-2,100-102$ & 38.61 & 42.13 & 42.13 & 2.50 & 25.87 & 6.52 & 27.00 & 25.30 & 70.50 & 63.29 \\
\hline $5 \mathrm{H}-4,100-102$ & 41.61 & 45.13 & 45.13 & 8.08 & 16.97 & 9.69 & 21.39 & 27.61 & 70.53 & 55.37 \\
\hline $6 \mathrm{H}-1,30-32$ & 45.91 & 49.98 & 49.98 & 0.33 & 25.57 & 12.77 & 30.63 & 27.16 & 69.04 & 54.39 \\
\hline $6 \mathrm{H}-6,145-150$ & 54.58 & 58.64 & 58.64 & 0.92 & 22.34 & 14.80 & 18.87 & 25.69 & 80.21 & 59.72 \\
\hline $9 \mathrm{H}-6,101-103$ & 82.62 & 90.63 & 90.63 & 15.50 & 35.08 & 17.64 & $\begin{array}{l}17.01 \\
17.92\end{array}$ & 23.64 & 66.58 & 58.92 \\
\hline $10 \mathrm{H}-2,101-103$ & 86.12 & 94.60 & 94.60 & 10.92 & 18.06 & 5.41 & 19.92 & 25.90 & 69.16 & 63.03 \\
\hline $10 \mathrm{H}-4,100-102$ & 89.11 & 97.60 & 97.60 & 4.25 & -1.06 & 6.64 & 21.90 & 23.78 & 73.85 & 68.27 \\
\hline $10 \mathrm{H}-6,100-102$ & 92.11 & 100.60 & 100.60 & 26.71 & 17.80 & 19.55 & 18.02 & 22.09 & 55.27 & 62.38 \\
\hline $11 \mathrm{H}-3,100-102$ & 97.11 & 106.88 & 106.88 & 0.17 & 17.59 & -2.88 & 10.00 & 23.33 & 89.83 & 67.10 \\
\hline $11 \mathrm{H}-5,100-102$ & 100.11 & 109.88 & 109.88 & 13.67 & 8.20 & 10.59 & 38.01 & 24.59 & 48.32 & 61.17 \\
\hline $12 \mathrm{H}-1,100-102$ & 103.61 & 114.95 & 114.95 & 12.92 & 8.04 & 4.10 & 32.33 & 24.13 & 54.75 & 66.79 \\
\hline $12 \mathrm{H}-3,100-102$ & 106.61 & 117.92 & 117.92 & 21.67 & 23.08 & 8.93 & 34.99 & 24.40 & 43.34 & 56.95 \\
\hline $12 \mathrm{H}-5,100-102$ & 109.61 & 120.95 & 120.95 & 16.00 & 1.29 & 1.70 & 28.93 & 24.53 & 55.07 & 66.70 \\
\hline $13 \mathrm{H}-1,100-102$ & 113.11 & 125.72 & 125.72 & 12.17 & 1.13 & 1.22 & 15.50 & 23.59 & 72.33 & 69.59 \\
\hline $13 \mathrm{H}-3,100-102$ & 116.11 & 128.73 & 128.73 & 0.25 & 4.26 & -0.47 & 25.02 & 22.33 & 74.73 & 68.17 \\
\hline $13 \mathrm{H}-6,100-102$ & 120.61 & 133.23 & 133.23 & 0.25 & 16.58 & -0.28 & 30.02 & 22.52 & 69.73 & 69.71 \\
\hline $14 \mathrm{H}-3,100-102$ & 125.61 & 139.60 & 139.60 & 0.50 & 22.29 & -0.65 & 17.10 & 20.58 & 82.40 & 74.89 \\
\hline $14 \mathrm{H}-5,100-102$ & 128.61 & 142.60 & 142.60 & 0.42 & 10.08 & 0.51 & 26.66 & 20.03 & 72.92 & 76.92 \\
\hline $15 \mathrm{H}-1,108-110$ & 132.19 & 148.06 & 148.06 & 0.42 & 6.75 & -1.57 & 11.32 & 20.29 & 88.26 & 83.88 \\
\hline $15 \mathrm{H}-4,108-110$ & 136.69 & 152.56 & 152.56 & 2.42 & 27.54 & 30.94 & 33.01 & 32.86 & 64.57 & 23.79 \\
\hline $15 \mathrm{H}-6,108-110$ & 139.69 & 155.56 & 155.56 & 21.42 & 21.71 & 25.31 & 17.37 & 32.86 & 61.21 & 41.21 \\
\hline $16 \mathrm{H}-2,110-112$ & 143.21 & 160.93 & 160.93 & 67.84 & 58.82 & 40.41 & 8.91 & 33.26 & 23.25 & 41.28 \\
\hline $16 \mathrm{H}-4,110-112$ & 146.21 & 163.93 & 163.93 & 46.42 & 18.49 & 37.19 & 16.97 & 27.02 & 36.61 & 39.18 \\
\hline $16 \mathrm{H}-6,110-112$ & 149.21 & 166.93 & 166.93 & 38.63 & 35.20 & 51.02 & 17.03 & 21.37 & 44.34 & 28.11 \\
\hline $17 \mathrm{H}-2,101-103$ & 152.62 & 171.43 & 171.43 & 80.17 & 73.16 & 78.01 & 5.68 & 4.03 & 14.15 & 14.88 \\
\hline $17 \mathrm{H}-4,101-103$ & 155.62 & 174.43 & 174.43 & 63.84 & 60.48 & 64.42 & 10.47 & 8.80 & 25.69 & 26.15 \\
\hline $17 \mathrm{H}-6,101-103$ & 158.62 & 177.43 & 177.43 & 77.34 & 67.95 & 71.76 & 4.66 & 7.28 & 18.00 & 26.93 \\
\hline $18 \mathrm{H}-2,100-102$ & 162.11 & 181.40 & 181.40 & 74.59 & 56.16 & 66.23 & 5.78 & 9.89 & 19.63 & 29.39 \\
\hline $18 \mathrm{H}-5,100-102$ & 166.61 & 185.90 & 185.90 & 75.59 & 67.75 & 75.77 & 5.03 & 7.67 & 19.38 & 17.95 \\
\hline $19 \mathrm{H}-1,101-103$ & 170.12 & 190.78 & 190.78 & 68.51 & 53.76 & 63.49 & 10.85 & 12.08 & 20.64 & 24.05 \\
\hline $19 \mathrm{H}-3,99-10 \mathrm{I}$ & 173.10 & 193.74 & 193.74 & 85.42 & 75.79 & 95.55 & 2.62 & 5.19 & 11.96 & -0.85 \\
\hline $19 \mathrm{H}-5,101-103$ & 176.12 & 196.78 & 196.78 & 83.17 & 82.50 & 88.05 & 6.05 & 7.97 & 10.78 & 2.31 \\
\hline $20 \mathrm{H}-1,103-105$ & 179.64 & 201.97 & 201.97 & 82.59 & 67.41 & 66.25 & 8.53 & 12.86 & 8.88 & 22.26 \\
\hline $20 \mathrm{H}-3,100-102$ & 182.61 & 204.95 & 204.95 & 79.17 & 82.38 & 79.15 & 9.96 & 11.13 & 10.87 & 11.84 \\
\hline $20 \mathrm{H}-5,100-102$ & 185.61 & 207.95 & 207.95 & 74.34 & 79.78 & 67.13 & 8.09 & 12.33 & 17.57 & 25.92 \\
\hline $21 \mathrm{H}-1,102-104$ & 189.13 & 212.35 & 212.35 & 75.92 & 76.92 & 85.01 & 8.13 & 8.42 & 15.95 & 9.15 \\
\hline $21 \mathrm{H}-3,99-101$ & 192.10 & 215.31 & 215.31 & 75.42 & 65.26 & 73.83 & 13.70 & 12.22 & 10.88 & 15.00 \\
\hline $21 \mathrm{H}-5,100-102$ & 195.11 & 218.35 & 218.35 & 79.59 & 76.79 & 72.72 & 8.70 & 11.85 & 11.71 & 16.51 \\
\hline
\end{tabular}


Table 2 (continued).

\begin{tabular}{|c|c|c|c|c|c|c|c|c|c|c|}
\hline $\begin{array}{l}\text { Core, section, } \\
\text { interval }(\mathrm{cm})\end{array}$ & $\begin{array}{l}\text { Depth } \\
\text { (mbsf) }\end{array}$ & $\begin{array}{l}\text { Depth } \\
\text { (mcd) }\end{array}$ & $\begin{array}{l}\text { Depth } \\
\text { (rmcd) }\end{array}$ & $\begin{array}{c}\mathrm{CaCO}_{3} \\
\text { (\% measured) }\end{array}$ & $\begin{array}{c}\mathrm{CaCO}_{3} \\
\text { (\% estimated from ( } \\
\text { GRAPE density) }\end{array}$ & $\begin{array}{l}\mathrm{CaCO}_{3} \\
\text { (\% estimated from } \\
\text { reflectance) }\end{array}$ & $\begin{array}{c}\text { Opal } \\
\text { (\% measured) }\end{array}$ & $\begin{array}{c}\text { Opal } \\
\text { (\% estimated from } \\
\text { reflectance) }\end{array}$ & $\begin{array}{l}\text { Nonbiogenic } \\
\text { (\% measured) }\end{array}$ & $\begin{array}{l}\text { Nonbiogenic } \\
\text { (\% estimated from } \\
\text { reflectance) }\end{array}$ \\
\hline $22 \mathrm{H}-1,101-103$ & 198.62 & 223.85 & 223.85 & 83.09 & 75.23 & 77.28 & 7.24 & 10.88 & 9.67 & 13.49 \\
\hline $22 \mathrm{H}-3,102-104$ & 201.63 & 226.85 & 226.85 & 89.76 & 88.40 & 90.41 & 3.71 & 7.43 & 6.53 & 2.26 \\
\hline $22 \mathrm{H}-5,100-102$ & 204.61 & 229.85 & 229.85 & 86.76 & 71.93 & 84.12 & 7.00 & 8.99 & 6.24 & 8.25 \\
\hline 138-846B- & & & & & & & & & & \\
\hline $1 \mathrm{H}-1,104-106$ & 1.05 & 1.06 & 1.06 & 60.71 & 44.17 & 50.87 & 33.52 & 50.68 & 5.77 & 8.86 \\
\hline $1 \mathrm{H}-3,104-106$ & 4.05 & 4.08 & 4.10 & 29.50 & 25.57 & 43.63 & 52.53 & 33.84 & 17.97 & 20.81 \\
\hline $1 \mathrm{H}-5,65-67$ & 6.66 & 6.45 & 6.33 & 58.67 & 36.38 & 55.67 & 37.09 & 36.72 & 4.24 & 9.04 \\
\hline $2 \mathrm{H}-2,103-105$ & 9.54 & 10.22 & 10.28 & 57.25 & 53.17 & 64.88 & 36.28 & 28.16 & 6.47 & 11.74 \\
\hline $2 \mathrm{H}-4,109-111$ & 12.60 & 13.22 & 13.24 & 72.17 & 63.40 & 59.90 & 22.58 & 35.28 & 5.25 & 11.86 \\
\hline $2 \mathrm{H}-6,103-105$ & 15.54 & 16.24 & 16.35 & 76.59 & 70.55 & 59.34 & 19.72 & 36.97 & 3.69 & 4.35 \\
\hline $3 \mathrm{H}-1,104-106$ & 17.55 & 19.60 & 19.58 & 73.59 & 75.32 & $\begin{array}{l}69.54 \\
69.55\end{array}$ & 19.11 & 26.46 & 7.30 & 6.44 \\
\hline $3 \mathrm{H}-3,104-106$ & 20.55 & 22.76 & 22.89 & 53.84 & 55.00 & 41.77 & 32.81 & 34.19 & 13.35 & 24.09 \\
\hline $3 \mathrm{H}-5,104-106$ & 23.55 & 25.62 & 25.62 & 64.76 & 64.99 & 58.37 & 27.30 & 35.28 & 7.94 & 5.05 \\
\hline $4 \mathrm{H}-1,103-105$ & 27.04 & 30.20 & 30.22 & 50.25 & 45.66 & 61.40 & 48.52 & 36.21 & 1.23 & 4.51 \\
\hline $4 \mathrm{H}-5,103-105$ & 33.04 & 36.06 & 35.98 & 53.67 & $\begin{array}{l}45.00 \\
66.58\end{array}$ & 55.42 & 20.09 & 30.31 & 26.24 & 12.39 \\
\hline $5 \mathrm{H}-1,104-106$ & 36.55 & 41.86 & 41.97 & $\begin{array}{l}53.01 \\
46.92\end{array}$ & 37.85 & $\begin{array}{l}59.82 \\
59.88\end{array}$ & $\begin{array}{l}20.09 \\
42.33\end{array}$ & $\begin{array}{l}30.31 \\
30.10\end{array}$ & 10.75 & 10.35 \\
\hline $5 \mathrm{H}-3,104-106$ & 39.55 & 44.66 & 44.55 & 37.34 & 52.19 & 38.39 & 45.05 & 32.39 & 17.61 & 26.89 \\
\hline $5 \mathrm{H}-5,104-106$ & 42.55 & 47.71 & 47.68 & $\begin{array}{l}58.67 \\
58.67\end{array}$ & 66.32 & $\begin{array}{l}37.43 \\
47.43\end{array}$ & 33.97 & $\begin{array}{l}32.39 \\
36.53\end{array}$ & $\begin{array}{r}1.01 \\
7.36\end{array}$ & 20.29 \\
\hline $6 \mathrm{H}-1,104-106$ & 46.05 & 52.56 & 52.56 & 54.92 & 47.38 & 62.45 & 36.79 & 27.68 & 8.29 & 2.81 \\
\hline $6 \mathrm{H}-5,103-105$ & 52.04 & 58.52 & 58.52 & 32.75 & 42.34 & 26.46 & 61.45 & 30.43 & 5.81 & 35.19 \\
\hline $7 \mathrm{H}-1,113-105$ & 55.64 & 63.79 & 63.64 & 48.00 & 47.05 & 37.16 & 43.42 & 33.42 & 8.58 & 28.54 \\
\hline $7 \mathrm{H}-3,104-106$ & 58.55 & 66.85 & 66.84 & 32.25 & 34.40 & 21.64 & 58.30 & 36.26 & $\begin{array}{l}.0 .00 \\
9.44\end{array}$ & 36.09 \\
\hline $7 \mathrm{H}-5,103-105$ & 61.54 & 69.82 & 69.82 & 14.42 & 26.05 & $\begin{array}{l}1.04 \\
19.00\end{array}$ & $\begin{array}{l}75.34 \\
75.30\end{array}$ & 36.73 & 10.24 & 34.72 \\
\hline $7 \mathrm{H}-7,38-40$ & $\begin{array}{l}63.89 \\
63.174\end{array}$ & 72.42 & 72.64 & 33.25 & 31.50 & 28.37 & 65.31 & 38.35 & 1.44 & 27.07 \\
\hline $8 \mathrm{H}-2,38-40$ & 65.89 & 75.53 & 75.51 & 35.59 & 34.11 & 34.54 & $\begin{array}{l}63.61 \\
63.51\end{array}$ & $\begin{array}{l}38.35 \\
38.04\end{array}$ & 0.80 & 21.84 \\
\hline $8 \mathrm{H}-6,90-92$ & 72.41 & 81.98 & 81.88 & 65.34 & 63.63 & 48.28 & & 40.44 & 4.88 & $\begin{array}{r}2.84 \\
4.23\end{array}$ \\
\hline $9 \mathrm{H}-3,104-106$ & 77.55 & 87.60 & 87.46 & 56.17 & 59.53 & 51.98 & 32.84 & 32.07 & 10.99 & 13.65 \\
\hline $9 \mathrm{H}-5,104-106$ & 80.55 & 90.70 & 90.69 & 65.92 & 65.29 & 49.72 & 24.85 & 36.59 & 9.23 & 7.53 \\
\hline $10 \mathrm{H}-1,107-109$ & 84.08 & 97.00 & 97.25 & 45.17 & 41.18 & 43.93 & 42.23 & $\begin{array}{l}44.09 \\
4.09\end{array}$ & 12.60 & 13.34 \\
\hline $10 \mathrm{H}-3,108-100$ & 87.09 & 99.67 & 99.67 & 45.67 & 52.86 & 60.26 & 43.01 & & 11.32 & 15.34 \\
\hline $10 \mathrm{H}-5,118-100$ & 90.19 & 102.71 & $\begin{array}{r}702.69 \\
109\end{array}$ & $\begin{array}{l}47.00 \\
47.00\end{array}$ & 54.95 & $\begin{array}{l}49.43 \\
49.43\end{array}$ & 47.63 & 37.20 & 5.37 & 12.97 \\
\hline $11 \mathrm{H}-1,97-99$ & 93.48 & 107.16 & 107.32 & 37.09 & 38.30 & 41.07 & 58.38 & 54.42 & 4.53 & 7.74 \\
\hline $11 \mathrm{H}-3,103-105$ & 96.54 & 110.34 & 110.66 & 65.92 & 79.38 & 67.80 & 18.12 & 17.86 & 15.96 & 7.22 \\
\hline $11 \mathrm{H}-5,103-105$ & 99.54 & 113.22 & 113.41 & 53.92 & 64.77 & 61.81 & 36.52 & 27.23 & 9.56 & 8.24 \\
\hline $12 \mathrm{H}-1,103-105$ & 103.04 & 117.89 & 117.97 & 56.42 & 60.80 & 69.21 & 33.05 & 18.28 & 10.53 & 8.58 \\
\hline $12 \mathrm{H}-3,103-105$ & 106.04 & 120.87 & 120.93 & 45.50 & 60.71 & 60.00 & 44.44 & 30.97 & 10.06 & 4.23 \\
\hline $12 \mathrm{H}-5,103-105$ & 109.04 & 123.83 & 123.84 & 42.50 & 58.59 & $\begin{array}{l}0.00 \\
48.68\end{array}$ & 37.64 & 29.47 & 19.86 & 17.49 \\
\hline $13 \mathrm{H}-1,102-104$ & 112.53 & 129.26 & 129.40 & 50.17 & 49.78 & $\begin{array}{l}+0.00 \\
63.78\end{array}$ & 41.28 & 25.84 & 8.55 & 8.69 \\
\hline $13 \mathrm{H}-3,101-103$ & 115.52 & 131.87 & 131.76 & 30.00 & 40.04 & 36.53 & 49.12 & 44.94 & 20.87 & $\begin{array}{r}0.09 \\
14.99\end{array}$ \\
\hline $13 \mathrm{H}-5,103-105$ & 118.54 & 135.36 & 135.86 & 57.34 & 69.66 & 56.84 & 30.60 & 33.68 & 12.06 & 5.34 \\
\hline $14 \mathrm{H}-1,102-104$ & 122.03 & 139.40 & 139.66 & 68.84 & 77.46 & 79.34 & 16.83 & 18.48 & 14.33 & 9.43 \\
\hline $14 \mathrm{H}-3,103-105$ & 125.04 & 142.33 & 142.47 & 72.01 & 78.80 & 73.48 & 16.59 & 20.21 & 11.40 & -0.49 \\
\hline $14 \mathrm{H}-5,135-107$ & 128.36 & 145.58 & 145.70 & 53.17 & 60.05 & 61.55 & 36.52 & 32.31 & 10.31 & 6.90 \\
\hline $15 \mathrm{H}-2,124-106$ & 133.25 & 152.96 & 152.96 & 34.42 & 57.86 & $\begin{array}{l}1.53 \\
40.58\end{array}$ & 43.15 & 29.46 & 22.43 & 32.49 \\
\hline $15 \mathrm{H}-4,103-105$ & 136.04 & 155.84 & 155.94 & 36.84 & $\begin{array}{l}78.99 \\
48.99\end{array}$ & 50.76 & 53.81 & 41.08 & 9.34 & 14.83 \\
\hline $15 \mathrm{H}-6,104-106$ & 139.05 & 158.81 & 158.85 & 58.09 & 69.14 & 52.68 & 22.75 & 28.13 & 19.16 & $\begin{array}{l}17.53 \\
17.03\end{array}$ \\
\hline $16 \mathrm{H}-2,102-104$ & 142.53 & 162.78 & 162.83 & 78.17 & 81.11 & 74.40 & 12.89 & 18.57 & 8.94 & 11.22 \\
\hline $16 \mathrm{H}-5,102-104$ & 147.03 & 167.18 & 167.15 & 76.67 & 81.03 & 76.28 & 11.36 & 19.03 & 11.97 & 5.10 \\
\hline $17 \mathrm{H}-1,102-104$ & 150.53 & 171.78 & 171.80 & 71.34 & 76.85 & 74.24 & 15.50 & 19.21 & 13.16 & 4.57 \\
\hline $17 \mathrm{H}-3,102-104$ & 153.53 & 174.76 & 174.75 & 67.05 & 65.16 & 58.27 & 27.06 & & 5.89 & 8.87 \\
\hline $17 \mathrm{H}-5,104-106$ & 156.55 & 177.91 & 178.01 & 44.21 & $\begin{array}{l}65.10 \\
65.08\end{array}$ & 59.20 & $\begin{array}{l}2.000 \\
46.58\end{array}$ & $\begin{array}{l}2.47 \\
32.81\end{array}$ & 9.21 & $\begin{array}{l}8.07 \\
8.11\end{array}$ \\
\hline $18 \mathrm{H}-1,110-102$ & 160.11 & 181.63 & 181.32 & 61.92 & 59.12 & 71.41 & $\begin{array}{l}40.58 \\
28.08\end{array}$ & 20.88 & $\begin{array}{r}3.21 \\
10.00\end{array}$ & $\begin{array}{l}6.90 \\
6.90\end{array}$ \\
\hline $18 \mathrm{H}-3,102-104$ & 163.03 & 184.92 & 184.96 & 84.59 & 93.17 & 83.84 & 8.81 & 10.44 & 6.60 & 6.21 \\
\hline $18 \mathrm{H}-5,102-104$ & 166.03 & 187.86 & 187.85 & 84.51 & 88.32 & 71.69 & 9.69 & 7.75 & $\begin{array}{l}0.00 \\
5.80\end{array}$ & 23.98 \\
\hline $19 \mathrm{H}-1,103-105$ & 169.54 & 193.39 & 193.49 & 78.26 & 81.87 & 70.39 & 12.89 & 20.58 & 8.85 & 4.27 \\
\hline $19 \mathrm{H}-3,103-105$ & 172.54 & 196.32 & 196.32 & 71.84 & 81.82 & 67.12 & 10.40 & 27.39 & 17.76 & 2.36 \\
\hline $19 \mathrm{H}-5,103-105$ & 175.54 & 199.32 & 199.32 & 81.09 & $\begin{array}{l}87.27 \\
87.27\end{array}$ & 75.22 & 11.02 & 18.86 & 7.89 & 3.75 \\
\hline $20 \mathrm{H}-2,103-105$ & 180.54 & 206.09 & 206.08 & 77.92 & 87.20 & 68.56 & 11.97 & $\begin{array}{l}23.54 \\
23.50\end{array}$ & 10.11 & 4.51 \\
\hline $20 \mathrm{H}-4,103-105$ & 183.54 & 209.16 & 209.19 & 71.26 & $\begin{array}{l}8.20 \\
71.43\end{array}$ & $\begin{array}{l}68.56 \\
70.41\end{array}$ & $\begin{array}{l}11.91 \\
19.55\end{array}$ & 19.53 & $\begin{array}{r}10.11 \\
9.19\end{array}$ & 7.77 \\
\hline $20 \mathrm{H}-6,103-105$ & 186.54 & 212.07 & 212.01 & 76.09 & 80.27 & 75.91 & 13.40 & 16.93 & 10.51 & 5.02 \\
\hline $21 \mathrm{H}-2,103-105$ & 190.04 & 216.97 & 216.97 & 69.01 & 72.91 & 66.32 & 25.09 & 31.02 & 5.90 & 1.08 \\
\hline $21 \mathrm{H}-4,103-105$ & 193.04 & 219.97 & 219.97 & 72.67 & 81.44 & 69.73 & 17.17 & 21.86 & 10.16 & 8.75 \\
\hline $21 \mathrm{H}-6,103-105$ & 196.04 & 222.96 & 222.95 & 73.42 & 87.92 & 76.38 & 16.83 & 20.71 & 9.75 & 7.51 \\
\hline $22 \mathrm{H}-2,103-105$ & 199.54 & 229.99 & 230.00 & 68.34 & 74.27 & 72.98 & 20.67 & 20.57 & 10.99 & 4.55 \\
\hline $22 \mathrm{H}-4,103-105$ & 202.54 & 232.97 & 232.97 & 63.59 & $\begin{array}{l}63.82 \\
63.27\end{array}$ & $\begin{array}{l}72.98 \\
73.83\end{array}$ & 28.08 & 21.46 & 8.33 & 3.97 \\
\hline $22 \mathrm{H}-6,103-105$ & 205.54 & 235.99 & 236.02 & 51.84 & 65.62 & 70.60 & 40.83 & 21.91 & 7.33 & 5.48 \\
\hline 138-847B- & & & & & & & & & & \\
\hline $\begin{array}{l}1 \mathrm{H}-2,102-104 \\
\end{array}$ & 2.53 & 2.52 & 2.52 & 39.00 & 24.91 & 40.49 & 41.00 & 21.35 & 20.00 & 33.33 \\
\hline $1 \mathrm{H}-4,103-105$ & 5.54 & 5.66 & 5.76 & 54.17 & 48.17 & 63.21 & 32.57 & 18.71 & 13.26 & 13.68 \\
\hline $2 \mathrm{H}-1,103-105$ & 7.54 & 7.64 & 7.63 & 50.17 & $\begin{array}{l}61.09 \\
61.17\end{array}$ & $\begin{array}{l}75.36 \\
75.21\end{array}$ & 30.67 & 17.37 & 19.16 & 5.12 \\
\hline $2 \mathrm{H}-3,32-34$ & 9.83 & 9.97 & 9.97 & 76.55 & 64.90 & 73.03 & 10.27 & 17.85 & 13.18 & 2.62 \\
\hline $2 \mathrm{H}-5,107-109$ & 13.58 & 13.69 & 13.69 & 79.59 & 79.36 & 77.40 & 13.26 & 12.00 & 7.15 & 4.77 \\
\hline $2 \mathrm{H}-7,14-16$ & 15.65 & 15.66 & 15.58 & 66.51 & 54.60 & 69.32 & 12.65 & 12.83 & 20.84 & 14.82 \\
\hline $3 \mathrm{H}-3,102-104$ & 20.03 & 20.35 & 20.33 & 80.09 & 76.39 & 70.40 & 12.38 & 19.39 & 7.53 & 7.90 \\
\hline $3 \mathrm{H}-5,102-104$ & 23.03 & 23.35 & 23.33 & 48.21 & 62.61 & 52.56 & 19.45 & 18.82 & 32.34 & 24.60 \\
\hline $3 \mathrm{H}-7,33-35$ & 25.34 & 25.57 & 25.43 & 58.00 & 47.50 & 69.84 & 20.16 & 13.58 & 21.84 & 12.60 \\
\hline $4 \mathrm{H}-2,102-104$ & 28.03 & 30.85 & 30.85 & 73.76 & 58.46 & $\begin{array}{l}62.04 \\
62.02\end{array}$ & 24.21 & 17.80 & 2.03 & 16.49 \\
\hline $4 \mathrm{H}-4,102-104$ & 31.03 & 33.65 & 33.56 & 58.34 & $\begin{array}{l}62.40 \\
62.38\end{array}$ & $\begin{array}{l}0.02 \\
54.56\end{array}$ & 22.17 & 22.63 & 19.49 & 17.79 \\
\hline $4 \mathrm{H}-6,102-104$ & 34.03 & 36.92 & 37.01 & 62.25 & 64.22 & 67.83 & 15.44 & 13.89 & 22.31 & 16.08 \\
\hline $5 \mathrm{H}-1,133-105$ & 36.34 & 39.14 & 39.14 & 69.34 & 66.03 & 73.62 & 13.91 & 15.17 & 16.75 & 5.25 \\
\hline $5 \mathrm{H}-3,104-106$ & 39.05 & 41.86 & 41.86 & 52.25 & 44.63 & 58.50 & 40.29 & 17.93 & 7.46 & 19.78 \\
\hline $5 \mathrm{H}-7,49-51$ & 44.50 & 47.30 & 47.30 & 57.34 & 57.96 & 66.49 & 33.46 & 19.61 & 9.20 & 9.37 \\
\hline $6 \mathrm{H}-2,103-105$ & 47.04 & 52.00 & 51.91 & 51.50 & 41.89 & 37.08 & 33.97 & 29.61 & 14.53 & 26.06 \\
\hline $6 \mathrm{H}-4,32-34$ & 49.33 & 54.32 & 54.23 & 25.29 & 20.05 & 13.78 & 45.66 & 26.36 & 29.05 & 50.95 \\
\hline $6 \mathrm{H}-6,104-106$ & 53.05 & 58.23 & 58.34 & 30.50 & 44.14 & 18.10 & 32.95 & 27.91 & 36.55 & 45.44 \\
\hline $7 \mathrm{H}-1,103-105$ & 55.04 & 61.78 & 61.77 & 52.75 & 44.07 & 41.58 & 30.12 & 27.45 & 17.13 & 25.09 \\
\hline
\end{tabular}


Table 2 (continued).

\begin{tabular}{|c|c|c|c|c|c|c|c|c|c|c|}
\hline $\begin{array}{l}\text { Core, section, } \\
\text { interval }(\mathrm{cm})\end{array}$ & $\begin{array}{l}\text { Depth } \\
\text { (mbsf) }\end{array}$ & $\begin{array}{l}\text { Depth } \\
\text { (mcd) }\end{array}$ & $\begin{array}{l}\text { Depth } \\
\text { (rmcd) }\end{array}$ & $\begin{array}{c}\mathrm{CaCO}_{3} \\
\text { (\% measured) }\end{array}$ & $\begin{array}{c}\mathrm{CaCO}_{3} \\
\text { (\% estimated from } \\
\text { GRAPE density) }\end{array}$ & $\begin{array}{l}\mathrm{CaCO}_{3} \\
\text { (\% estimated from } \\
\text { reflectance) }\end{array}$ & $\begin{array}{c}\text { Opal } \\
\text { (\% measured) }\end{array}$ & $\begin{array}{c}\text { Opal } \\
\text { (\% estimated from } \\
\text { reflectance) }\end{array}$ & $\begin{array}{l}\text { Nonbiogenic } \\
\text { (\% measured) }\end{array}$ & $\begin{array}{c}\text { Nonbiogenic } \\
\text { (\% estimated from } \\
\text { reflectance) }\end{array}$ \\
\hline $7 \mathrm{H}-3,103-105$ & 58.04 & 64.63 & 64.46 & 26.59 & 31.05 & 10.02 & 50.25 & 28.95 & 23.16 & 51.13 \\
\hline $7 \mathrm{H}-5,103-105$ & 61.04 & 67.45 & 67.15 & 59.00 & 59.52 & 61.84 & 31.45 & 20.78 & 9.55 & 13.82 \\
\hline $7 \mathrm{H}-7,37-39$ & 63.38 & 70.13 & 70.13 & 68.34 & 72.28 & 57.86 & 21.22 & 19.33 & 10.44 & 18.07 \\
\hline $8 \mathrm{H}-2,103-105$ & 66.04 & 74.13 & 74.07 & 69.09 & 68.83 & 67.37 & 19.48 & 22.71 & 11.43 & 4.10 \\
\hline $8 \mathrm{H}-4,103-105$ & 69.04 & 77.26 & 77.31 & 60.17 & 57.21 & 64.14 & 25.91 & 16.97 & 13.92 & 16.46 \\
\hline $8 \mathrm{H}-6,100-102$ & 72.01 & 80.39 & 80.59 & 73.34 & 78.16 & 82.76 & 16.42 & 10.35 & 10.24 & 4.18 \\
\hline $9 \mathrm{H}-2,26-28$ & 74.77 & 84.20 & 84.15 & 40.78 & 40.89 & 44.80 & 44.68 & 25.88 & 14.54 & 22.06 \\
\hline $9 \mathrm{H}-4,103-105$ & 78.54 & 87.87 & 87.71 & 77.17 & 68.51 & 53.25 & 12.44 & 21.84 & 10.39 & 17.87 \\
\hline $9 \mathrm{H}-6,97-99$ & 81.48 & 91.00 & 91.03 & 70.51 & 77.96 & 75.40 & 16.76 & 15.38 & 12.73 & 2.86 \\
\hline $10 \mathrm{H}-2,109-110$ & 85.10 & 95.11 & 94.97 & 67.34 & 62.79 & 56.85 & 14.69 & 17.46 & 17.97 & 20.82 \\
\hline $10 \mathrm{H}-4,104-106$ & 88.05 & 98.29 & 98.29 & 74.59 & 81.82 & 76.21 & 16.05 & 12.79 & 9.36 & 7.52 \\
\hline $10 \mathrm{H}-6,102-104$ & 91.03 & 101.25 & 101.25 & 66.51 & 66.39 & 65.51 & 19.52 & 16.43 & 13.97 & 11.83 \\
\hline $1 \mathrm{H}-1,32-34$ & 92.33 & 105.02 & 104.84 & 61.25 & 62.58 & 63.97 & 24.11 & 19.07 & 14.64 & 8.20 \\
\hline $11 \mathrm{H}-3,32-34$ & 95.33 & 108.13 & 108.04 & 62.42 & 68.08 & 65.10 & 23.80 & 18.11 & 13.78 & 9.36 \\
\hline $11 \mathrm{H}-5,34-36$ & 98.35 & 111.20 & 111.15 & 68.01 & 76.15 & 72.98 & 14.45 & 12.20 & 17.54 & 10.96 \\
\hline $12 \mathrm{H}-1,33-35$ & 101.84 & 114.82 & 114.76 & 72.67 & 64.24 & 66.63 & 13.60 & 15.88 & 13.73 & 12.01 \\
\hline $12 \mathrm{H}-3,33-35$ & 104.84 & 117.78 & 117.70 & 65.17 & 64.15 & 48.67 & 18.33 & 19.46 & 16.50 & 24.51 \\
\hline $12 \mathrm{H}-5,33-35$ & 107.84 & 120.74 & 120.66 & 67.09 & 64.07 & 64.80 & 17.68 & 15.56 & 15.23 & 16.65 \\
\hline $12 \mathrm{H}-7,44-46$ & 110.95 & 124.05 & 124.10 & 76.01 & 82.63 & 67.12 & 12.24 & 17.54 & 11.75 & 12.05 \\
\hline $13 \mathrm{H}-2,33-35$ & 112.84 & 126.85 & 126.86 & 68.34 & 63.93 & 43.83 & 18.67 & 23.21 & 12.99 & 26.32 \\
\hline $13 \mathrm{H}-4,33-35$ & 115.84 & 129.87 & 129.90 & 81.51 & 81.30 & 70.80 & 8.60 & 11.50 & 9.89 & 14.65 \\
\hline $13 \mathrm{H}-6,33-35$ & 118.84 & 132.86 & 132.88 & 65.17 & 70.93 & 40.98 & 15.27 & 18.33 & 19.56 & 38.25 \\
\hline $14 \mathrm{H}-1,33-35$ & 120.84 & 136.41 & 136.50 & 74.13 & 83.64 & 81.26 & 8.53 & 9.10 & 17.34 & 5.82 \\
\hline $14 \mathrm{H}-3,33-35$ & 123.84 & 139.26 & 139.18 & 74.67 & 81.15 & 77.06 & 14.21 & 12.90 & 11.12 & 5.91 \\
\hline $14 \mathrm{H}-5,102-104$ & 127.53 & 143.04 & 143.09 & 42.92 & 53.23 & 55.51 & 43.66 & 25.26 & 13.42 & 15.77 \\
\hline $14 \mathrm{H}-7,33-35$ & 129.84 & 145.30 & 145.29 & 61.09 & 75.46 & 65.93 & 28.90 & 23.61 & 10.01 & 9.95 \\
\hline $15 \mathrm{H}-2,33-35$ & 131.84 & 146.15 & 145.61 & 56.21 & 67.12 & 65.18 & 30.97 & 25.02 & 12.82 & 8.48 \\
\hline $15 \mathrm{H}-5,33-35$ & 136.34 & 147.66 & 146.11 & 46.00 & 50.71 & 54.19 & 41.39 & 35.70 & 12.61 & 13.07 \\
\hline $15 \mathrm{H}-7,33-35$ & 139.34 & 148.66 & 146.44 & 31.79 & 57.17 & 48.12 & 49.47 & 26.90 & 18.74 & 18.98 \\
\hline
\end{tabular}

chosen to exclude disturbed intervals around sediment biscuits, the estimates of $\mathrm{CaCO}_{3}$ percentage from GRAPE were more precise (RMS error $\pm 11.8 \%, r^{2}=0.74$ ) than the estimates from reflectance (RMS error $\pm 14.3 \%, r^{2}=0.62$ ). Note that the GRAPE estimates include corrections for downhole compaction and porosity rebound. The reflectance estimates were calibrated only with unlithified samples from APC cores and contain no corrections for compaction. We conclude that errors in reflectance associated either with surface roughness or with lithification in XCB cores degrade the predictions of lithology slightly. Improvements may come from (1) calibrating equations to predict lithologies from reflectance in partially lithified materials separately from unlithified materials, (2) improving methods of splitting partially lithified sediment cores to minimize surface roughness, or (3) reducing the sensitivity to surface roughness by measuring diffuse, rather than directional, reflectance.

To further check the fidelity of the two estimates of $\mathrm{CaCO}_{3}$ concentration within the APC cores, we compare the statistical properties of the reflectance and GRAPE estimates in Site 846, over the same time series represented in Figure 7. The distribution of variance in this time series is essentially identical for reflectance and GRAPE estimates. The power spectra demonstrate that both estimators have similar distributions of variance over the last $3.5 \mathrm{Ma}$, with significant concentrations in the primary earth orbital bands of 41,23 , and 19 k.y. and at long periods (Fig. 9A). The two time series are significantly coherent (Fig. 9B) and in phase (Fig. 9C) in essentially all bands in which the data variance is large.

The horizontal dashed line in Figure 9A represents the noise level in calibration of $\mathrm{CaCO}_{3}$ percentages based on GRAPE density. The noise level for the reflectance estimate would be slightly lower. This calculation of noise level in variance density units assumes that misfits are random (white) noise that can be represented by the total variance $\left(\sigma^{2}\right)$ times the fraction of variance left unexplained $\left(1-r^{2}\right)$, divided by the bandwidth of this analysis $\left(0.0012\right.$ k.y. $\left.{ }^{-1}\right)$. Where variance in the time series of the GRAPE and reflectance estimates of $\mathrm{CaCO}_{3}$ concentration drops below that expected for noise in the calibration (at high frequencies), the two calcium carbonate estimates are not coherent, and we cannot assume that either is a good estimate of true concentration of $\mathrm{CaCO}_{3}$. The strong coherency of the two estimates at longer periods, however, indicates that both are recording similar lithologic variations for the frequency bands in which the variance is strong. Because the assumptions behind the GRAPE and reflectance methods are completely different, we concluded that both are good measures of the history of $\mathrm{CaCO}_{3}$ concentration in the eastern tropical Pacific Ocean.

\section{SUMMARY AND CONCLUSIONS}

Reflectance spectroscopy has great potential for providing long, high-resolution time series of lithologic proxy measurements at a precision sufficient for many studies of marine geologic history. The technique can be used to scan unprepared core surfaces. It is rapid enough that large data sets can be produced at sea as cores are recovered and opened. Quantitative estimates of lithology can be calibrated with reasonably small ground-truth data sets generated by traditional chemical methods. This technique is efficient in both cost and materials, as it works well with automation, minimizes laborintensive chemical measurements, and preserves material for other measurements that must consume sediment.

Reflectance measurements performed during Leg 138 were calibrated here to estimate quantitatively the concentrations of $\mathrm{CaCO}_{3}$, opal, and nonbiogenic sediment components in the eastern tropical Pacific Ocean. The equations calibrated here are not universal, but are applicable within the geologic setting of the eastern Pacific. Estimates of $\mathrm{CaCO}_{3}$ percentage from reflectance spectra are robust over a large range, with RMS errors of about $9 \%$. This precision is as good or better than carbonate estimates based on GRAPE density measurements, in environments having a significant nonbiogenic component. Reflectance spectroscopy will be especially useful in these environments. Estimates of the concentration of nonbiogenic sediment are usually reasonable, but not as successful as the estimates of $\mathrm{CaCO}_{3}$ percentage. Mismatches between the estimates of nonbiogenic sediment made by reflectance and chemical measurements may point to variations in the mineralogy of the nonbiogenic sediment that warrant further study. Estimates of opal concentration may work reasonably well at low opal concentrations, but are poorly constrained at high concentrations. 

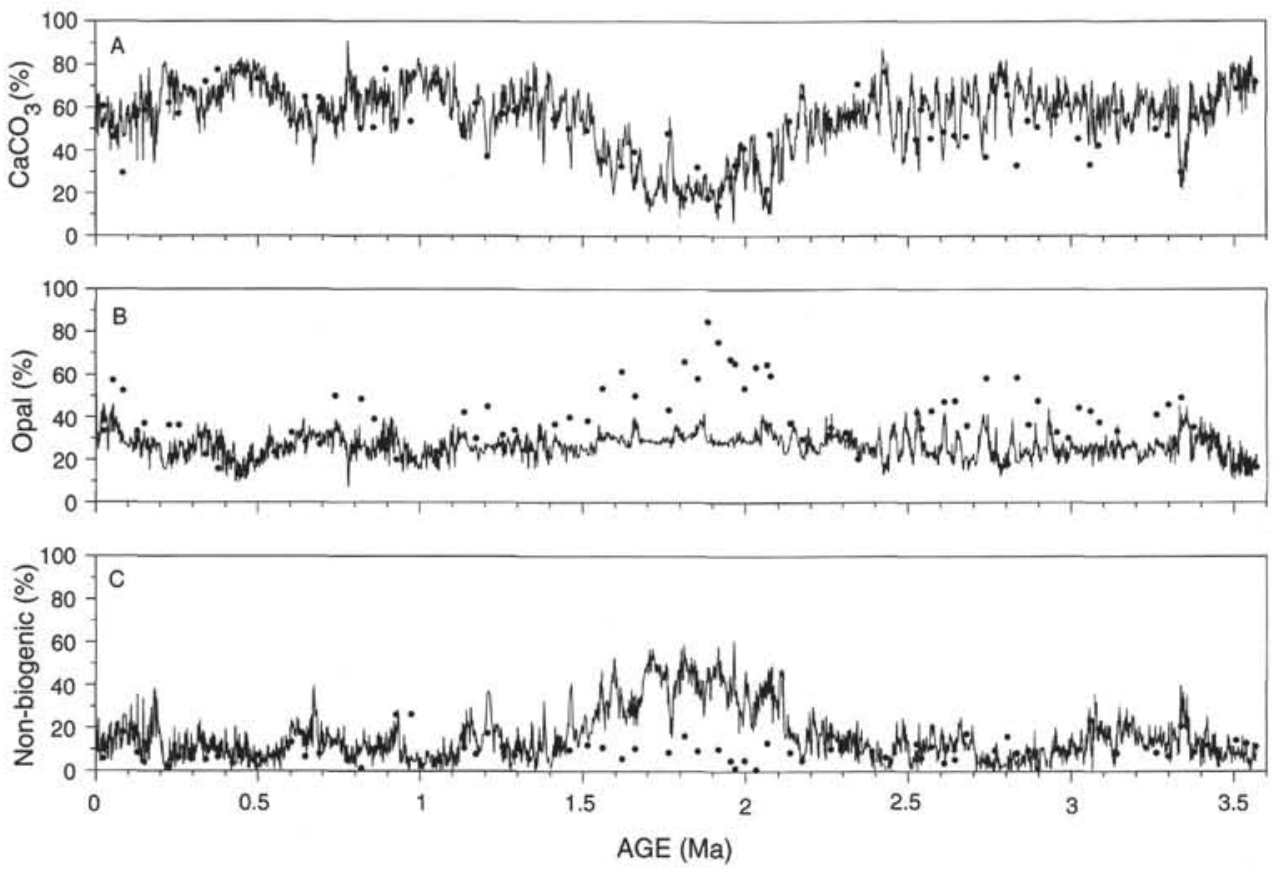

Figure 7. Time series reconstructions of sedimentary components at Site 846 , estimated from reflectance spectra. A. CaCO $\%$. B. Opal\%. C. Nonbiogenic sediment\%. Estimated $\mathrm{CaCO}_{3}$ and nonbiogenic sediment percentages are strongly negatively correlated $(r=-0.95)$. Estimated opal is weakly correlated to nonbiogenic sediment percentages $(r=+0.61)$. Solid circles are discrete ground-truth samples measured chemically. In $\mathbf{B}$, reflectance often underestimates high opal concentrations, producing a signal of artificially low amplitude. In $\mathbf{C}$, the estimates of nonbiogenic sediment are generally good, but overestimate values from 1.5 to $2.1 \mathrm{Ma}$.
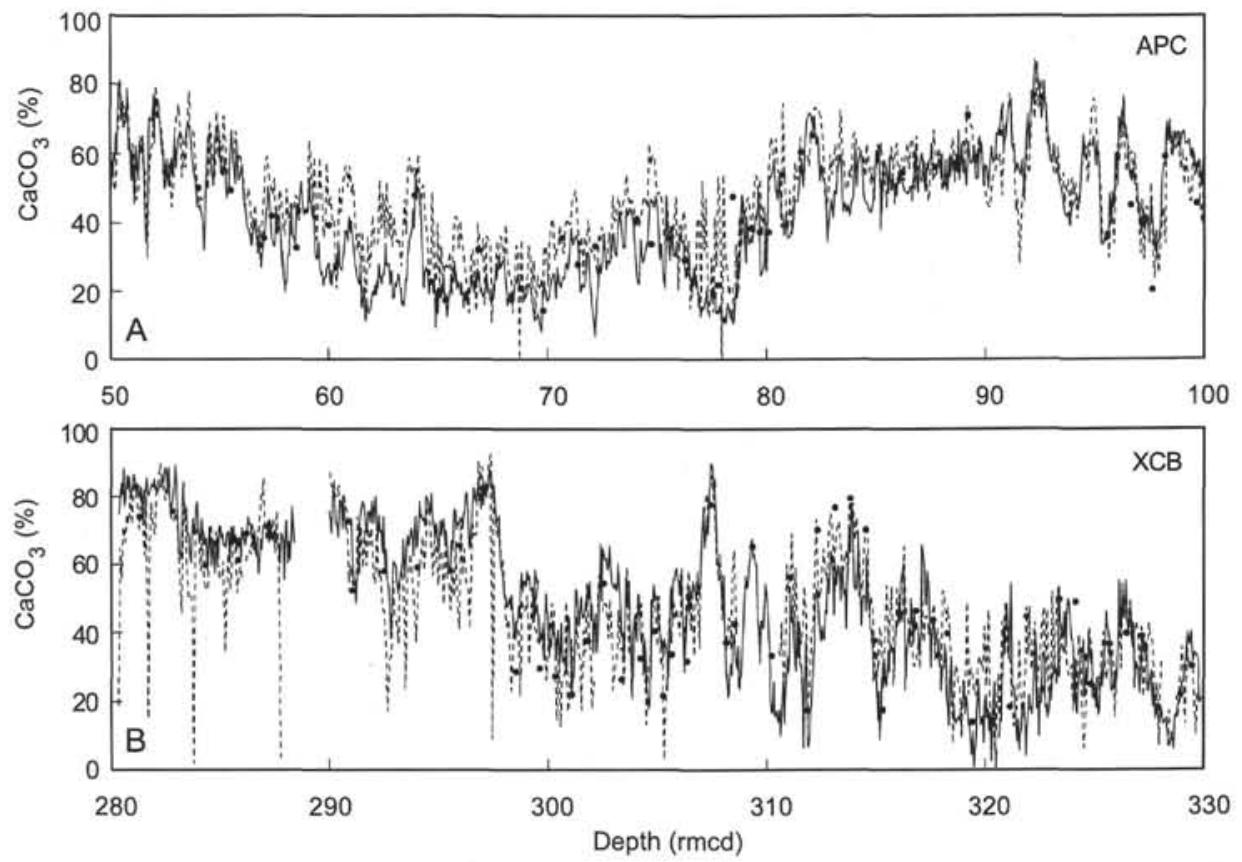

Figure 8. Percentages of $\mathrm{CaCO}_{3}$ from Site 846, comparing estimates from reflectance spectra (solid line), from GRAPE density (dashed line), and from discrete chemical measurements (solid points) in typical intervals of APC and XCB cores. A. Example of APC cores from Holes 846B, 846C, and 846D, plotted as a complete record from depths of 50 to 100 rmcd in the "shipboard splice," a composite record that fills gaps between cores with complete intervals from adjacent holes (Hagelberg et al., 1992). The sample interval for the reflectance and GRAPE estimates is $4 \mathrm{~cm}$. B. Example of XCB cores from Hole 846B, from depths of 280-330 rmcd. No attempt was made to create a composite section by combining data from multiple holes at these depths. The sample interval for reflectance measurements was $8 \mathrm{~cm}$. GRAPE data were interpolated to $8-\mathrm{cm}$ intervals at approximately the same sample depths as the reflectance data. Note the low spikes in GRAPE density estimates of $\mathrm{CaCO}_{3}$ percentage in the XCB interval. These are probably an artifact of GRAPE density measurements, including disturbed, water-saturated material around oblong sediment "biscuits," common in XCB cores. Reflectance estimates, measured along the axis of the cores where biscuits tend to meet, are not sensitive to this error and thus may yield a better estimate of lithology in materials recovered by XCB. 
Table 3. Measured and estimated $\mathrm{CaCO}_{3}$ percentages in $\mathrm{XCB}$ cores.

\begin{tabular}{|c|c|c|c|c|c|c|c|c|c|}
\hline $\begin{array}{l}\text { Core, section, } \\
\text { interval }(\mathrm{cm})\end{array}$ & $\begin{array}{l}\text { Depth } \\
\text { (mbsf) }\end{array}$ & $\begin{array}{c}\mathrm{CaCO}_{3} \\
(\% \text { measured })\end{array}$ & $\begin{array}{l}\mathrm{CaCO}_{3} \\
(\%)^{\mathrm{a}}\end{array}$ & $\begin{array}{l}\mathrm{CaCO}_{3} \\
(\%)^{\mathrm{b}}\end{array}$ & $\begin{array}{l}\text { Core, section, } \\
\text { interval }(\mathrm{cm})\end{array}$ & $\begin{array}{l}\text { Depth } \\
\text { (mbsf) }\end{array}$ & $\begin{array}{c}\mathrm{CaCO}_{3} \\
(\% \text { measured })\end{array}$ & $\begin{array}{r}\mathrm{CaCO}_{3} \\
(\%)^{\mathrm{a}}\end{array}$ & $\begin{array}{r}\mathrm{CaCO}_{3} \\
(\%)^{\mathrm{b}}\end{array}$ \\
\hline 138-844B- & & & & & $32 X-4,105-107$ & 298.66 & 7.83 & 22.14 & 10.40 \\
\hline $21 X-6,100-102$ & 193.51 & 74.30 & 70.54 & 43.16 & $32 X-5,106-108$ & 300.17 & 5.92 & -2.94 & -4.18 \\
\hline $30 \mathrm{X}-2,107-109$ & 274.18 & 72.80 & 85.41 & 39.98 & $32 X-6,96-98$ & 301.57 & 34.00 & 27.71 & 32.28 \\
\hline $30 X-4,82-84$ & 267.93 & 56.60 & 76.52 & 45.95 & $33 \mathrm{X}-1,103-105$ & 303.84 & 22.25 & 38.31 & 37.11 \\
\hline $30 X-6,106-108$ & 280.17 & 64.30 & 80.60 & 44.89 & $34 \mathrm{X}-3,123-125$ & 316.64 & 50.09 & 49.96 & 36.00 \\
\hline $31 X-2,125-127$ & 283.96 & 51.30 & 62.76 & 28.59 & $34 X-4,113-115$ & 318.04 & 33.09 & 42.82 & 35.42 \\
\hline 138-846B- & & & & & $34 X-5,33-35$ & 318.74 & 59.00 & 62.55 & 59.22 \\
\hline $23 \mathrm{X}-1,103-105$ & 207.54 & 71.59 & 67.38 & 59.91 & $34 \mathrm{X}-7,23-25$ & 321.64 & 68.26 & 66.23 & 59.89 \\
\hline $23 \mathrm{X}-2,103-105$ & 209.04 & 68.17 & 78.48 & 68.31 & $35 X-4,119-121$ & 327.70 & 64.76 & 74.50 & 58.00 \\
\hline $23 \mathrm{X}-3,103-105$ & 210.54 & 73.26 & 77.03 & 63.11 & $35 X-5,114-116$ & 329.15 & 55.92 & 60.29 & 39.34 \\
\hline $23 \mathrm{X}-4,103-105$ & 212.04 & 73.51 & 78.42 & 77.20 & $35 X-6,114-116$ & 330.65 & 67.92 & 71.23 & 62.36 \\
\hline $23 X-5,103-105$ & 213.54 & 64.67 & 76.97 & 68.59 & $36 \mathrm{X}-1,108-110$ & 332.79 & 65.01 & 62.16 & 31.29 \\
\hline $23 \mathrm{X}-6,103-105$ & 215.04 & 75.42 & 88.57 & 69.73 & $36 \mathrm{X}-2,130-132$ & 334.51 & 72.92 & 49.39 & 58.52 \\
\hline $24 \mathrm{X}-1,102-104$ & 217.23 & 84.42 & 91.59 & 79.97 & $36 \mathrm{X}-3,130-132$ & 336.01 & 66.01 & 72.74 & 57.43 \\
\hline $24 X-2,102-104$ & 218.73 & 79.26 & 93.70 & 76.72 & $36 \mathrm{X}-4,122-124$ & 337.43 & 74.17 & 72.70 & 64.97 \\
\hline $24 \mathrm{X}-3,102-104$ & 220.23 & 82.92 & 86.62 & 72.52 & $36 \mathrm{X}-6,105-107$ & 340.26 & 63.51 & 49.20 & 55.08 \\
\hline $24 \mathrm{X}-4,102-104$ & 221.73 & 70.17 & 79.59 & 73.25 & $37 X-2,95-97$ & 343.76 & 85.09 & 84.74 & 73.43 \\
\hline $24 X-5,102-104$ & 223.23 & 67.92 & 86.58 & 69.29 & $38 \mathrm{X}-1,98-100$ & 351.99 & 62.25 & 55.48 & 68.49 \\
\hline $24 X-6,102-104$ & 224.73 & 76.26 & 86.56 & 65.71 & $38 X-5,114-116$ & 358.15 & 67.34 & 48.62 & 69.41 \\
\hline $25 \mathrm{X}-1,102-104$ & 226.73 & 61.34 & 38.53 & 65.34 & $40 \mathrm{X}-1,123-125$ & 371.54 & 79.42 & 83.15 & 48.22 \\
\hline $25 \mathrm{X}-2,103-105$ & 228.24 & 66.09 & 61.16 & 78.51 & $40 X-2,103-105$ & 372.84 & 84.26 & 92.03 & 53.62 \\
\hline $25 X-3,103-105$ & 229.74 & 67.59 & 68.59 & 59.49 & $40 X-3,105-107$ & 374.36 & 69.92 & 89.95 & 44.61 \\
\hline $25 \mathrm{X}-4,103-105$ & 231.24 & 75.59 & 79.40 & 72.05 & $40 X-4,88-90$ & 375.69 & 73.51 & 92.58 & 40.13 \\
\hline $27 \mathrm{X}-1,103-105$ & 246.24 & 74.09 & 58.63 & 75.85 & $40 X-5,120-122$ & 377.51 & 71.01 & 70.11 & 36.13 \\
\hline $27 \mathrm{X}-2,103-105$ & 247.74 & 75.67 & 81.69 & 65.00 & $41 X-1,102-104$ & 380.93 & 90.63 & 82.99 & 78.43 \\
\hline $27 \mathrm{X}-3,103-105$ & 249.24 & 59.75 & 62.53 & 63.89 & $41 X-3,115-117$ & 384.06 & 88.13 & 69.95 & 64.48 \\
\hline $27 X-4,103-105$ & 250.74 & 61.17 & 58.49 & 58.65 & $41 X-4,82-84$ & 385.23 & 81.34 & 69.93 & 67.79 \\
\hline $27 X-5,103-105$ & 252.24 & 68.26 & 62.45 & 64.38 & $42 X-1,91-93$ & 390.52 & 78.59 & 83.99 & 56.19 \\
\hline $28 \mathrm{X}-1,103-105$ & 255.94 & 52.42 & 54.11 & 67.41 & $44 X-2,130-132$ & 411.71 & 75.59 & 93.33 & 24.71 \\
\hline $28 \mathrm{X}-2,101-103$ & 257.42 & 58.00 & 47.27 & 54.00 & $44 X-4,94-96$ & 414.35 & 66.17 & 72.56 & 35.88 \\
\hline $28 \mathrm{X}-3,102-104$ & 258.93 & 59.09 & 51.81 & 57.99 & 138-847B- & & & & \\
\hline $28 X-4,103-105$ & 260.44 & 58.00 & 56.12 & 59.12 & $22 X-5,33-35$ & 199.54 & 71.92 & 87.65 & 73.81 \\
\hline $28 \mathrm{X}-5,103-105$ & 261.94 & 82.51 & 82.66 & 81.36 & $22 \mathrm{X}-6,32-34$ & 201.03 & 56.59 & 73.86 & 62.82 \\
\hline $28 \mathrm{X}-6,103-105$ & 263.44 & 28.71 & 23.56 & 35.30 & $22 X-7,23-25$ & 202.44 & 71.09 & 83.41 & 73.43 \\
\hline $29 \times-1,103-105$ & 265.24 & 27.42 & 26.33 & 34.96 & $23 \mathrm{X}-1,31-33$ & 203.12 & 83.09 & 86.64 & 86.86 \\
\hline $29 \mathrm{X}-2,102-104$ & 266.73 & 37.67 & 51.56 & 46.93 & $23 \mathrm{X}-2,32-34$ & 204.63 & 74.34 & 80.92 & 72.02 \\
\hline $29 \mathrm{X}-3,108-110$ & 268.29 & 26.50 & 37.02 & 35.85 & $23 \times-3,31-33$ & 206.12 & 76.92 & 86.59 & 72.71 \\
\hline $29 X-4,104-106$ & 269.75 & 40.59 & 53.68 & 48.87 & $23 X-4,32-34$ & 207.63 & 75.26 & 91.52 & 69.15 \\
\hline $29 X-5,102-104$ & 271.23 & 31.75 & 46.81 & 41.92 & $23 \mathrm{X}-5,32-34$ & 209.13 & 67.17 & 86.55 & 70.27 \\
\hline $29 \times-6,63-65$ & 272.34 & 77.80 & 77.18 & 76.55 & $23 \mathrm{X}-6,33-35$ & 210.64 & 59.75 & 27.82 & 64.56 \\
\hline $29 X-7,22-24$ & 273.43 & 42.59 & 31.54 & 30.30 & $23 \mathrm{X}-7,22-24$ & 212.03 & 72.76 & 91.48 & 68.27 \\
\hline $30 X-2,58-60$ & 275.99 & 56.25 & 57.75 & 48.51 & $24 \mathrm{X}-1,32-34$ & 212.83 & 69.09 & 87.47 & 75.28 \\
\hline $30 \times-2,135-137$ & 276.76 & 17.50 & 28.67 & 3.81 & $24 \mathrm{X}-2,32-34$ & 214.33 & 75.26 & 80.73 & 79.00 \\
\hline $30 X-3,112-114$ & 278.03 & 76.76 & 67.35 & 49.55 & $24 X-3,32-34$ & 215.83 & 72.84 & 75.07 & 65.62 \\
\hline $30 X-4,102-104$ & 279.43 & 70.17 & 61.69 & 42.64 & $24 \mathrm{X}-4,32-34$ & 217.33 & 81.59 & 86.43 & 82.98 \\
\hline $30 X-5,104-106$ & 280.95 & 45.42 & 55.49 & 37.89 & $24 \mathrm{X}-5,33-35$ & 218.84 & 84.76 & 94.00 & 79.67 \\
\hline $30 X-6,106-108$ & 282.47 & 43.67 & 51.06 & 37.69 & $24 X-6,32-34$ & 220.33 & 67.01 & 84.25 & 76.40 \\
\hline $30 \times-7,23-25$ & 283.14 & 39.75 & 25.63 & 19.23 & $25 \mathrm{X}-1,32-34$ & 222.53 & 85.17 & 92.01 & 84.19 \\
\hline $31 X-1,79-81$ & 284.30 & 14.08 & 25.58 & 7.04 & $25 \mathrm{X}-2,32-34$ & 224.03 & 67.17 & 73.33 & 67.96 \\
\hline $31 X-2,103-105$ & 286.04 & 18.42 & 38.95 & 45.10 & $25 \mathrm{X}-3,30-32$ & 225.51 & 75.67 & 80.52 & 71.63 \\
\hline $31 X-3,103-105$ & 287.54 & 23.34 & 57.41 & 31.47 & $25 \mathrm{X}-4,41-43$ & 227.12 & 64.34 & 71.64 & 62.74 \\
\hline $31 X-4,101-103$ & 289.02 & 48.92 & 25.39 & 31.71 & $25 \mathrm{X}-5,38-40$ & 228.59 & 69.01 & 74.79 & 78.54 \\
\hline $31 X-5,103-105$ & 290.54 & 36.67 & 38.78 & 33.55 & $25 X-6,31-33$ & 230.02 & 54.67 & 56.53 & 63.76 \\
\hline $31 X-6,103-105$ & 292.04 & 38.92 & 41.25 & 27.76 & $25 X-7,23-25$ & 231.44 & 57.25 & 66.33 & 66.75 \\
\hline \multirow{2}{*}{$\begin{array}{l}32 \mathrm{X}-2,103-105 \\
32 \mathrm{X}-3,106-108\end{array}$} & 295.64 & 24.42 & 16.36 & 10.29 & & & & & \\
\hline & 297.17 & 9.75 & 7.00 & 10.65 & 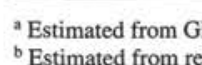 & & & & \\
\hline
\end{tabular}

The present study of Leg 138 sediments considered reflectance spectra over most of the visible and some of the nIR bands (455$945 \mathrm{~nm}$ ). The instrument was a first-generation prototype, and many improvements are expected in the future. We think that major improvements will come from (1) expansion of the wavelengths measured to better capture unique mineral reflectance signatures, (2) collection of diffuse, rather than directional, reflectance to minimize the effects of surface roughness, and (3) improvements in array detector technology that will improve the signal/noise ratios and speed up the analyses.

\section{ACKNOWLEDGMENTS}

We thank USSAC for funding of instrument development and post-cruise science, and the Leg 138 shipboard party for assistance at sea. This manuscript benefited from helpful reviews by W. Busch, S. O'Connell, and W. Balsam, and editorial comments from L. Mayer.

\section{REFERENCES}

Baker, E.Q., and Louda, J.W., 1986. Porphyrins in the geological record. In Johns, R.B. (Ed.) Biological Markers in the Sedimentary Record: Amsterdam (Elsevier), 125-225.

Balsam, W.L., 1992. Estimating sediment organic content and percent calcium carbonate in marine sediment from NUV/VIS/NIR spectra. Eos, 73:308.

Balsam, W.L., and Deaton, B.C., 1991. Sediment dispersal in the Atlantic Ocean: evaluation by visible light spectra. Rev. Aquat. Sci., 4:411-447.

Barranco, F.T., Jr., Balsam, W.L., and Deaton, B.C., 1989. Quantitative reassessment of brick red lutites: evidence from reflectance spectrophotometry. Mar. Geol., 89:299-314.

Abbreviations for names of organizations and publications in ODP reference lists follow
the style given in Chemical Abstracts Service Source Index (published by American Chemical Society). 
Boyce, R.E., 1976. Definitions and laboratory techniques of compressional sound velocity parameters and wet-water content, wet-bulk density, and porosity parameters by gravimetric and gamma ray attenuation techniques. In Schlanger, S.O., Jackson, E.D., et al., Init. Repts. DSDP, 33: Washington (U.S. Govt. Printing Office), 931-958.

Busch, W.H., 1991. Analysis of wet-bulk density and sediment color cycles in Pliocene-Pleistocene sediments of the Owen Ridge (Site 722) and Oman Margin (Site 728). In Prell, W.L., Niitsuma, N., et al., Proc. ODP, Sci. Results, 117: College Station, TX (Ocean Drilling Program), 239-253.

Clark, R.N., 1981. The spectral reflectance of water-mineral mixtures at low temperatures. J. Geophys. Res., 86:3074-3086.

Clark, R.N., Trude, V., King, V., Klejwa, M., Swayze, G.A., and Vergo, N., 1990. High spectral resolution reflectance spectroscopy of minerals. $J$. Geophys. Res., 95:12653-12680.

Deaton, B.C., and Balsam, W.L., 1991. Visible spectroscopy: a rapid method for determining hematite and goethite concentrations in geological materials. J. Sediment. Petrol., 61:629-632.

Gaffey, S.J., 1986. Spectral reflectance of carbonate minerals in the visible and near infrared $(0.35$ to $2.55 \mu \mathrm{m})$ : calcite, aragonite, and dolomite. Am. Mineral., 71:151-162.

Hagelberg, T., Shackleton, N., Pisias, N., and Shipboard Scientific Party, 1992. Development of composite depth sections for Sites 844 through 854. In Mayer, L., Pisias, N., Janecek, T., et al., Proc. ODP, Init. Repts., 138 (Pt. 1): College Station, TX (Ocean Drilling Program), 79-85.

Heath, G.R., Moore, T.C., Jr., and Roberts, G.L., 1974. Mineralogy of surface sediments from the Panama Basin, Eastern Equatorial Pacific. J. Geol., $82: 145-160$.

Herbert, T.D., and Mayer, L.A., 1991. Long climatic time series from sediment physical property measurements. J. Sediment. Petrol., 61:1089-1108.

Hoffer, R.M., 1984. Remote sensing to measure the distribution and structure of vegetation. I $n$ Woodwell, G.M. (Ed.), The Role of Terrestrial Vegetation in the Global Carbon Cycle: Measurement by Remote Sensing: New York (Wiley), 131-159.

Hunt, G.R., 1977. Spectral signatures of particulate minerals in the visible and near infrared. Geophysics, 42:501-513.

Hunt, G.R., and Salisbury, J.W., 1970. Visible and near-infrared spectra of minerals and rocks. I: Silicate minerals. Mod. Geol., 1:283-300.

, 1971. Visible and near-infrared spectra of minerals and rocks. II: Carbonates. Mod. Geol., 2:23-30.

Hunt, G.R., Salisbury, J.W., and Lenhoff, C.J., 1971a. Visible and near-infrared spectra of minerals and rocks. III: Oxides and hydroxides. Mod. Geol., 2:195-205.

, 1971b. Visible and near-infrared spectra of minerals and rocks. IV: Sulphides and sulphates. Mod. Geol., 3:1-14

Lyle, M., 1983. The brown-green color transition in marine sediments: a marker of the Fe(III)-Fe(II) redox boundary. Limnol. Oceanogr., 28:1026-1033.

Mayer, L.A., 1991. Extraction of high-resolution carbonate data for paleoclimate reconstruction. Nature, 352:148-150.

Mayer, L.A., Pisias, N.G., Janecek, T.R., et al., 1992. Proc. ODP, Init. Repts., 138 (Pts. 1 and 2): College Station, TX (Ocean Drilling Program).

Mix, A.C., Rugh, W., Pisias, N.G., Veirs, S., Leg 138 Shipboard Sedimentologists (Hagelberg, T., Hovan, S., Kemp, A., Leinen, M., Levitan, M., Ravelo, C.), and Leg 138 Scientific Party, 1992. Color reflectance spectroscopy: a tool for rapid characterization of deep-sea sediments. In Mayer, L., Pisias, N., Janecek, T., et al., Proc. ODP, Init. Repts., 138 (Pt. 1): College Station, TX (Ocean Drilling Program), 67-77.

Mortlock, R.A., and Froelich, P.N., 1989. A simple method for the rapid determination of biogenic opal in pelagic marine sediments. Deep-Sea Res. Part A, 36:1415-1426.

Schumann, D., and Nagel, U., 1982. Leg 68: shore-based X-ray mineralogy. In Prell, W.L., Gardner, J.V., et al., Init. Repts. DSDP, 68: Washington (U.S. Govt. Printing Office), 397-402.

Zimmerman, H.B., 1982. Lithologic stratigraphy and clay mineralogy of the western Caribbean and eastern equatorial Pacific, Leg 68, Deep Sea Drilling Project. In Prell, W.L., Gardner, J.V., et al., Init. Repts. DSDP, 68: Washington (U.S. Govt. Printing Office), 383-395.
Site $846,0-3.5 \mathrm{Ma}$
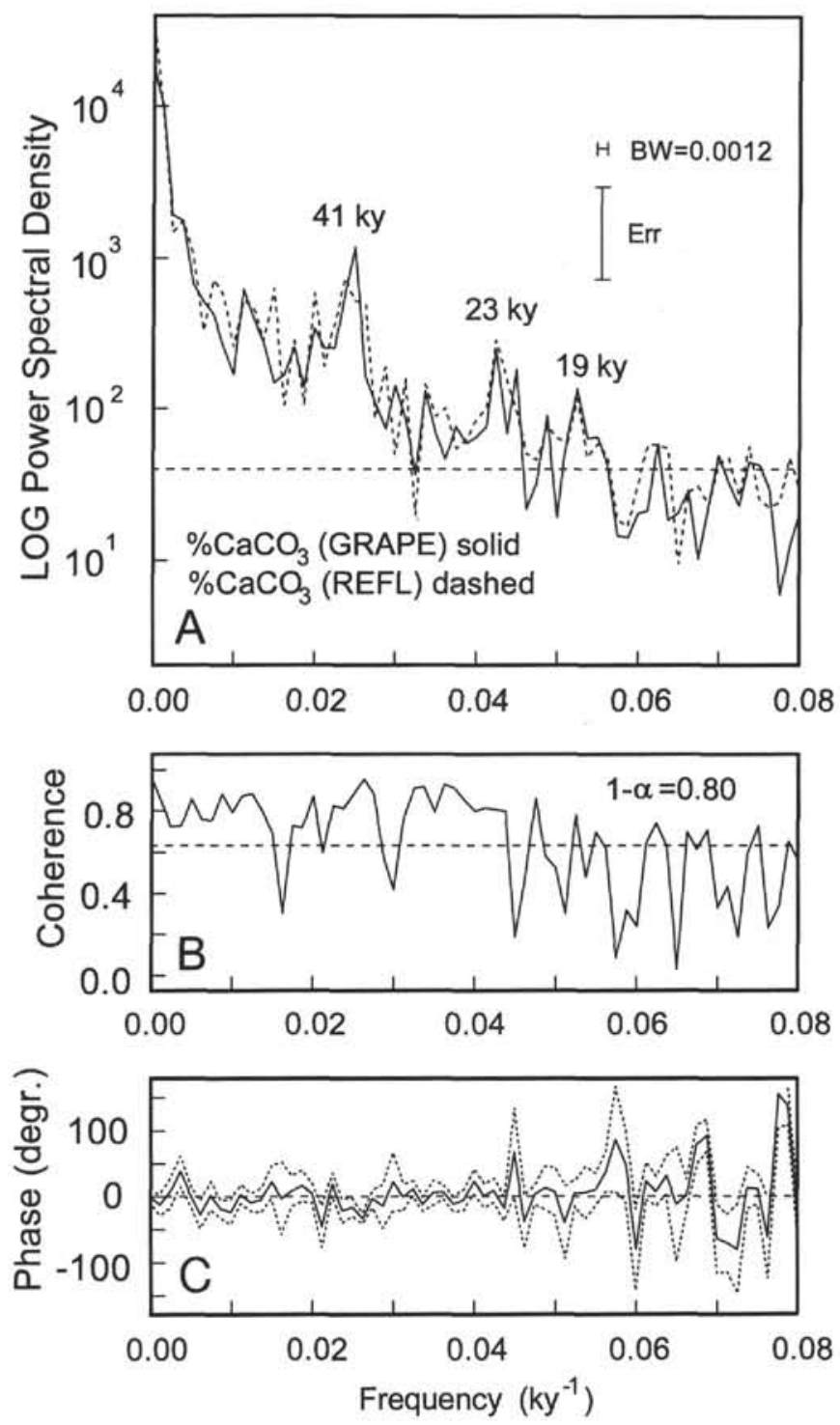

Figure 9. Time series analysis compares the estimates of $\mathrm{CaCO}_{3}$ percentage based on GRAPE density with those based on reflectance spectra over the past $3.5 \mathrm{Ma}$ at Site 846 . Bandwidth of this analysis is $0.0012 \mathrm{k} . \mathrm{y}^{-1}$. A. Power spectral density as a function of frequency demonstrates a similar distribution of variance in both estimates (GRAPE density is the solid line, reflectance is dashed). The horizontal dashed line is the noise level of the GRAPE calibration, assuming that all variance in the calibration data set for $\mathrm{CaCO}_{3}$ percentage unaccounted for by GRAPE estimates is "white" noise, distributed equally in all frequency bands. B. The coherency spectrum between GRAPE and reflectance estimates of $\mathrm{CaCO}_{3}$ indicates that both are linearly correlated with each other, at greater than $80 \%$ confidence interval $(1-\alpha>0.80)$ in essentially all frequency bands that contain significant variance $\left(<0.55 \mathrm{k} \cdot \mathrm{y}^{-1}\right)$. C. The phase spectrum between GRAPE and reflectance estimates of $\mathrm{CaCO}_{3}$ indicates that both are in phase with each other, within an error envelope of $80 \%$ confidence interval, in essentially all frequency bands that contain significant variance $\left(<0.55 \mathrm{k} . \mathrm{y}^{-1}\right)$. 